ARTICLE

\title{
Actin dynamics provides membrane tension to merge fusing vesicles into the plasma membrane
}

Peter J. Wen ${ }^{1, \star}$, Staffan Grenklo ${ }^{2,3, \star}$, Gianvito Arpino ${ }^{1,2}$, Xinyu Tan ${ }^{4}$, Hsien-Shun Liao ${ }^{5}$, Johanna Heureaux ${ }^{4}$, Shi-Yong Peng ${ }^{1}$, Hsueh-Cheng Chiang ${ }^{1, \dagger}$, Edaeni Hamid', Wei-Dong Zhao1, Wonchul Shin', Tuomas Näreoja ${ }^{2}$, Emma Evergren ${ }^{2} \dagger$, Yinghui Jin ${ }^{1}$, Roger Karlsson ${ }^{3}$, Steven N. Ebert ${ }^{6}$, Albert Jin ${ }^{5}$, Allen P. Liu ${ }^{4}$, Oleg Shupliakov ${ }^{2,7}$ \& Ling-Gang $\mathrm{Wu}^{1}$

Vesicle fusion is executed via formation of an $\Omega$-shaped structure ( $\Omega$-profile), followed by closure (kiss-and-run) or merging of the $\Omega$-profile into the plasma membrane (full fusion). Although $\Omega$-profile closure limits release but recycles vesicles economically, $\Omega$-profile merging facilitates release but couples to classical endocytosis for recycling. Despite its crucial role in determining exocytosis/endocytosis modes, how $\Omega$-profile merging is mediated is poorly understood in endocrine cells and neurons containing small $\sim 30-300 \mathrm{~nm}$ vesicles. Here, using confocal and super-resolution STED imaging, force measurements, pharmacology and gene knockout, we show that dynamic assembly of filamentous actin, involving ATP hydrolysis, N-WASP and formin, mediates $\Omega$-profile merging by providing sufficient plasma membrane tension to shrink the $\Omega$-profile in neuroendocrine chromaffin cells containing $\sim 300 \mathrm{~nm}$ vesicles. Actin-directed compounds also induce $\Omega$-profile accumulation at lamprey synaptic active zones, suggesting that actin may mediate $\Omega$-profile merging at synapses. These results uncover molecular and biophysical mechanisms underlying $\Omega$-profile merging.

\footnotetext{
${ }^{1}$ National Institute of Neurological Disorders and Stroke, 35 Convent Drive, Building 35, Room 2B-1012, Bethesda, Maryland 20892, USA. ${ }^{2}$ Center of Excellence in Developmental Biology, Department of Neuroscience, Karolinska Institutet, S-171 77 Stockholm, Sweden. ${ }^{3}$ Department of Cell Biology, WGI, Stockholm University, 10691 Stockholm, Sweden. ${ }^{4}$ Department of Mechanical Engineering, University of Michigan, Ann Arbor, Michigan 48109, USA. ${ }^{5}$ National Institute of Biomedical Imaging and Bioengineering (NIBIB), Bethesda, Maryland 20892, USA. ${ }^{6}$ Burnett School of Biomedical Sciences, College of Medicine, University of Central Florida, 6900 Lake Nona Boulevard, Orlando, Florida 32827, USA. ${ }^{7}$ Institute of Translational Biomedicine, St. Petersburg State University, St. Petersburg 199034, Russia. ${ }^{*}$ These authors contributed equally to this work. † Present addresses: Department of Pharmacology, College of Medicine, National Cheng Kung University, No.1, University Road, Tainan city, Taiwan 701 (H.-C.C.); Centre for Cancer Research and Cell Biology, Queen's University Belfast, Belfast BT9 7AE, UK (E.E.). Correspondence and requests for materials should be addressed to O.S. (email: oleg.shupliakov@ki.se) or to L.-G.W. (email: wul@ninds.nih.gov).
} 
$\mathrm{V}$ esicle fusion releases vesicular contents such as hormones, peptides and transmitters, to mediate many biological processes crucial to an animal's life, such as stress responses, mood changes, synaptic transmission, neuronal network activity, and immune responses ${ }^{1-4}$. It is executed via formation of an $\Omega$-shape intermediate structure, termed $\Omega$-profile, at the plasma membrane for releasing contents, followed by closure (called kiss-and-run) or merging of the $\Omega$-profile into the plasma membrane (called full fusion) ${ }^{1-4}$. $\Omega$-profile closure limits vesicular content release and cargo delivery, but recycles vesicles economically ${ }^{1,2}$. In contrast, $\Omega$-profile merging allows for rapid, complete content release and cargo delivery, but couples exocytosis to classical endocytosis involving membrane invagination, $\Omega$-profile formation and fission, for retrieving merged vesicles ${ }^{1-3}$. In other words, $\Omega$-profile merging defines the mode of fusion (full fusion versus kiss-andrun) and the mode of endocytosis (classical endocytosis versus kiss-and-run). Despite these fundamental roles, the mechanism underlying $\Omega$-profile merging is unclear in endocrine cells and neurons where vesicles are $\leq \sim 300 \mathrm{~nm}$ and fusion takes place rapidly after calcium influx. $\Omega$-profile merging is often assumed to be a passive, automatic process with no energy consumption once fusion pore opens in neurons and neuroendocrine cells.

$\Omega$-profile merging has recently been studied in secretory cells containing extremely large vesicles $(\sim 1-5 \mu \mathrm{m})$, such as in Xenopus oocytes ${ }^{5}$, human endothelial cells, lacrimal epithelial acinar cells $s^{6}$, parotid and pancreatic acinar cells ${ }^{7,8}$, and type II pneumocytes ${ }^{9}$, where $\Omega$-profile merging and release take extremely long time (tens of seconds to tens of minutes) and release is not as tightly coupled to calcium influx as in neuroendocrine cells and neurons (reviewed in ref. 10). These studies suggest that cytoskeletal filamentous actin (F-actin) may coat the fusing $\Omega$-profile in a few to tens of seconds after fusion, which may mediate two seemingly opposite functions: to compress the fusing $\Omega$-profile and thus to merge the $\Omega$-profile with the plasma membrane, or to hold the $\Omega$-profile from collapsing into the plasma membrane. Whether and which of these mechanisms apply to endocrine cells and neurons containing smaller vesicles remain unclear, mostly due to difficulty of detecting the very transient process of $\Omega$-profile merging in smaller vesicles.

In the present work, we overcame the difficulty of detecting $\Omega$-profile merging in smaller vesicles by confocal imaging and super-resolution stimulated emission depletion (STED) imaging in neuroendocrine chromaffin cells ${ }^{11}$ and by electron microscopy (EM) at lamprey synapses. Combined with pharmacological tools and gene knockout (KO), we found that dynamic assembly of cytoskeletal F-actin is necessary for $\Omega$-profile merging in chromaffin cells. With imaging and manipulations of plasma membrane mechanical forces, our results suggest that F-actin mediates $\Omega$-profile merging by providing sufficient tension at the plasma membrane to shrink the $\Omega$-profile. We also found that the F-actin assembly pathway including hydrolysis of the energy molecule ATP, neuronal Wiskott-Aldrich syndrome protein (NWASP) and formin that activate F-actin assembly participates in mediating $\Omega$-profile merging. F-actin-mediated $\Omega$-profile merging is probably applicable to synapses, because block of F-actin led to accumulation of $\Omega$-profiles at the active zone of lamprey giant synapses. These results uncover novel molecular and biophysical mechanisms underlying $\Omega$-profile merging in neuroendocrine cells and neurons, which mediates full fusion and couples exocytosis to classical endocytosis.

\section{Results}

Imaging in conditions that facilitate $\boldsymbol{\Omega}$-profile merging. We used a recently developed technique to image $\Omega$-profile merging in live, primary-cultured bovine adrenal chromaffin cells containing $\sim 300 \mathrm{~nm}$ dense-core vesicles in a bath solution containing membrane-impermeable Alexa Fluor 647 (A647) and Alexa Fluor 488 (A488) (Fig. 1a) ${ }^{11}$. Cells were voltage clamped at $-80 \mathrm{mV}$ and stimulated with 10 pulses of $50 \mathrm{~ms}$ depolarization to $+10 \mathrm{mV}$ at $2 \mathrm{~Hz}\left(\operatorname{Train}_{2 \mathrm{~Hz}}\right)$. The resulting calcium current (ICa) and capacitance increases that reflect fusion were whole-cell recorded (Fig. 1b). During and within $3 \mathrm{~s}$ after Train ${ }_{2 \mathrm{~Hz}}, \mathrm{~A} 647$ and A488 spots reflecting dye-filled fusion-generated $\Omega$-profiles appeared $^{11}$ (Fig. 1c-f).

Consistent with our previous findings using a $1 \mathrm{~s}$ depolarization $^{11}, \Omega$-profiles may change in seven patterns (Supplementary Fig. 1a), which are grouped into three categories (Fig. 1c) as follows: (1) the $\Omega$-profile shrinks until undetectable, leading to $\Omega$-profile merging with the plasma membrane, termed $\Omega$-shrink fusion; (2) the $\Omega$-profile is maintained while its size may remain unchanged, enlarged or shrink to some extent, termed stay fusion; and (3) the stay fusion is followed by pore closure, termed close fusion ${ }^{11}$. With confocal imaging of A647 and A488 (every $20-40 \mathrm{~ms})$ at the cell bottom using strong and weak excitation, respectively (confocal A647 strong $/ \mathrm{A} 488_{\text {weak }}$ setting) ${ }^{11}, \Omega$-shrink was identified as parallel decay of A647 and A488 spot fluorescence $\left(F_{647}\right.$ and $F_{488}$, respectively) to baseline, whereas A647 (or A488) spot size, measured from the full-width halfmaximum $\left(W_{\mathrm{H}}\right)$, reduced until below confocal resolution (Fig. 1d and Supplementary Fig. 1b,c; for detail, see ref. 11). Stay fusion was identified as the persistence of the A647 and A488 spot for $>30$ s (our recording time; Fig. 1e). During stay fusion, the spot's $W_{\mathrm{H}}$ may remain unchanged, reduce to some extent or enlarge, whereas $F_{647}$ and $F_{488}$ changed in parallel (Supplementary Fig. 1d; for detail, see ref. 11). Close fusion was reflected as stay fusion followed by bleaching of the spot $F_{647}$ to baseline, but no change of $F_{488}$ signal (Fig. If and Supplementary Fig. 1e), because pore closure prevented exchange of bleached A647 (due to strong excitation) with fluorescent A647 in the bath, resulting in the decay of the spot $F_{647}$, but not the weakly excited $F_{488}$ (ref. 11). These three categories were confirmed with several other methods, including high-resolution STED microscopy (for example, Supplementary Fig. 1c) ${ }^{11}$.

With $1 \mathrm{~s}$ depolarization, we previously found that $\Omega$-shrink fusion percentage increases as ICa decreases ${ }^{11}$. To search for stimulation protocols that facilitate $\Omega$-shrink fusion, we decreased ICa charge by reducing the depolarization duration to $50 \mathrm{~ms}$. To induce more granule fusion, we used 10 pulses of $50 \mathrm{~ms}$ depolarization at $2 \mathrm{~Hz}\left(\operatorname{Train}_{2 \mathrm{~Hz}}\right)$. With $\operatorname{Train}_{2 \mathrm{~Hz}}, 68 \pm 4 \%$ of fusion events were $\Omega$-shrink fusion, $29 \pm 3 \%$ were stay fusion and $3 \pm 2 \%$ were close fusion (Fig. $1 \mathrm{~g}, \mathrm{~h} ; n=18$ cells, total fusion spots: 192; \pm indicates s.e. throughout this study). Similarly, whole-cell dialysis of $1.5 \mu \mathrm{M}$ free calcium induced mostly $\Omega$-shrink fusion (Fig. 1i). Throughout this study, we used $\operatorname{Train}_{2 \mathrm{~Hz}}$ to induce fusion if not mentioned otherwise.

Dynamic F-actin assembly mediates $\Omega$-profile merging. Application of latrunculin A (Lat A, $3 \mu \mathrm{M}$, bath), which disrupted F-actin polymerization ${ }^{12}$, reduced $\Omega$-shrink fusion from 68 to $19 \%$ ( 13 cells), increased stay fusion from 29 to $76 \%$, but did not affect close fusion (Fig. 2a,b). The remaining $\Omega$-shrink fusion in the presence of Lat $\mathrm{A}$ had a longer $F_{647}$ decay time constant $(\tau, 2.8 \mathrm{~s}$, for example, Fig. 2c; summarized in Fig. $2 \mathrm{~d}$ ) than in control $(1.2 \mathrm{~s}$, for example, Fig. $1 \mathrm{~d}, \mathrm{~g})$, indicating a reduction in $\Omega$-profile shrinking rate. Thus, Lat A slows down or blocks $\Omega$-profile shrinking. The percentage decrease in $\Omega$-shrink fusion was mirrored by the increased stay fusion percentage (Fig. 2b), indicating that Lat A converts $\Omega$-shrink to stay fusion. Similarly, another actin polymerization inhibitor cytochalasin D (Cyto D, 
$4 \mu \mathrm{M}$, bath $)^{13,14}$ inhibited $\Omega$-shrink and increased stay fusion, suggesting that actin polymerization mediates $\Omega$-profile merging (Fig. 2b,d and Supplementary Fig. 2). As expected ${ }^{12-14}$, Lat A and Cyto D significantly reduced F-actin labelled with overexpressed Lifeact-TagGFP2 (Lifeact) at the cell bottom where we examined fusion (Fig. 2e,f).

Similar to Lat A/Cyto D, wiskostatin $(10 \mu \mathrm{M})$, which inhibits N-WASP-mediated, Arp2/3-dependent F-actin assembly ${ }^{15}$, and SMIFH2 $(25 \mu \mathrm{M})$, which blocks formin-profilin binding involved in formin-dependent F-actin assembly ${ }^{16}$, inhibited $\Omega$-shrink and promoted stay fusion (Fig. 2g). In contrast, nocodazole, a microtubule assembly blocker, had no effects $(n=9$ cells, $10 \mu \mathrm{M})$. These results suggest that F-actin assembly pathways are involved in mediating $\Omega$-profile merging. Interestingly, whole-cell dialysis of phalloidin-fluorescein isothiocyanate (FITC; $1.3 \mu \mathrm{M}, 2-3 \mathrm{~min}$ ), which stabilizes F-actin, inhibited $\Omega$-shrink fusion, promoted stay fusion (Supplementary Fig. 3a,b), but did not affect the Lifeact intensity at the cell bottom (Supplementary Fig. 3c,d). These results suggest that dynamic F-actin polymerization is needed to mediate $\Omega$-profile merging.

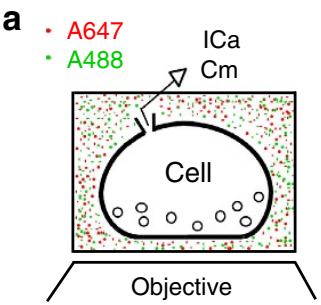

d
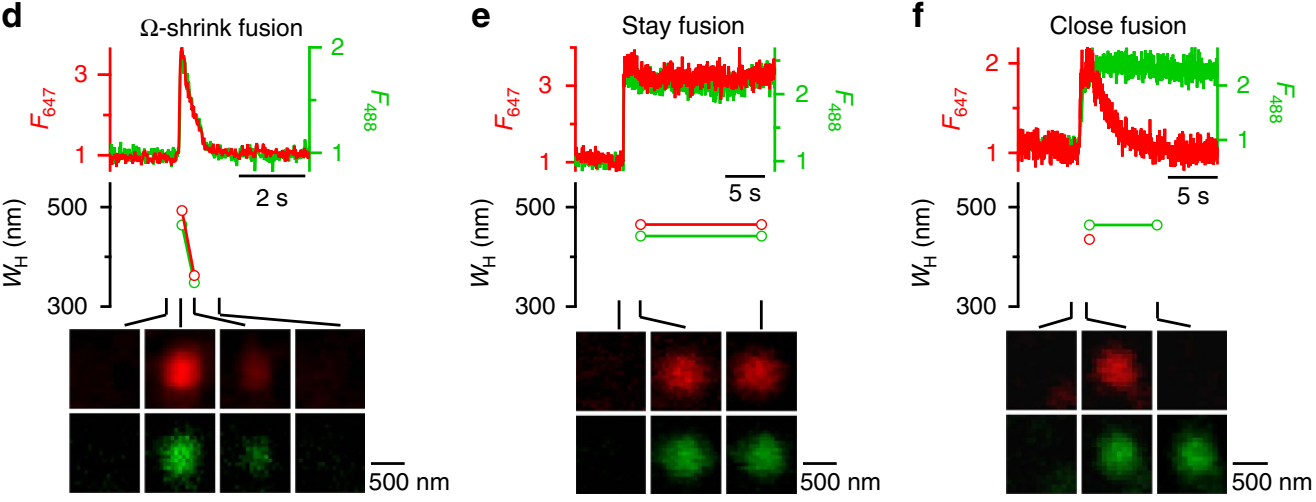

g

g Spot 1: $\Omega$-shrink fusion
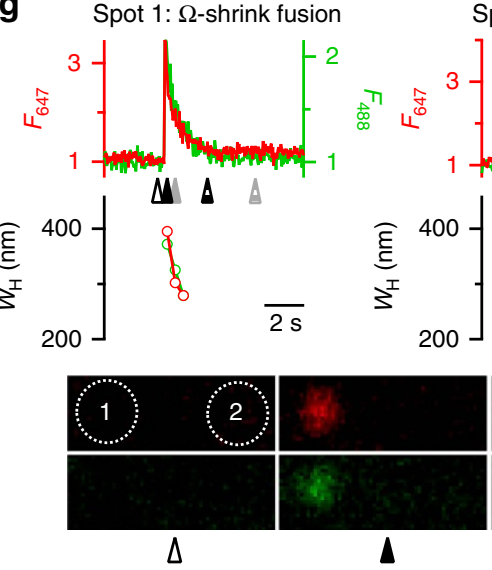

Spot 2: $\Omega$-shrink fusion

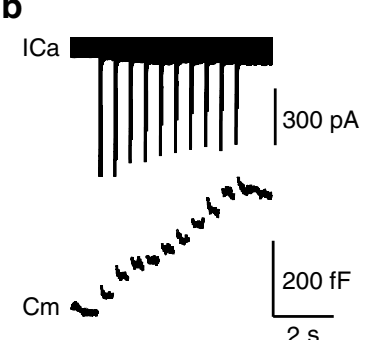

C

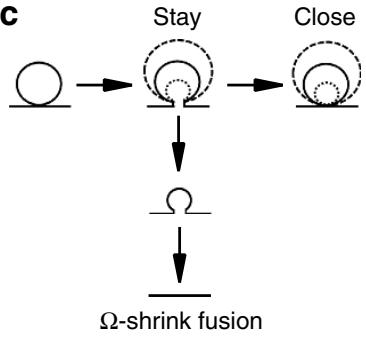

h

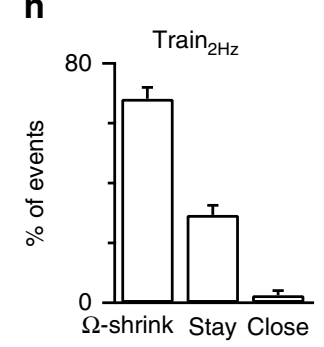

i

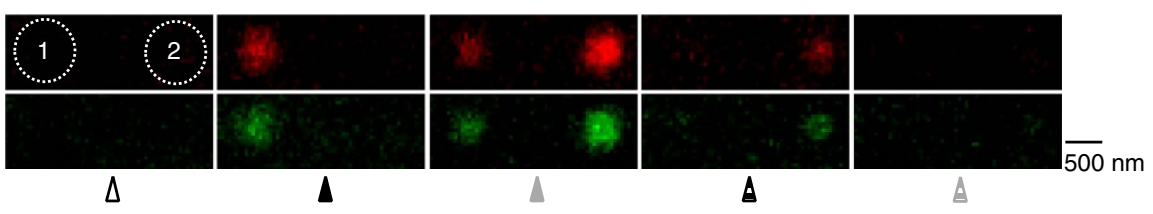

Figure 1 | Train ${ }_{2 \mathrm{~Hz}}$ induces three fusion forms with $\mathbf{\Omega}$-shrink as the dominant form in control chromaffin cells. (a) Schematic drawing of a cell on the coverslip bathed with a solution containing A647 (red) and A488 (green). ICa and membrane capacitance ( $\mathrm{Cm}$ ) are whole-cell recorded and the cell bottom is imaged confocally. (b) Sampled ICa and the $\mathrm{Cm}$ change induced by $\operatorname{Train}_{2 \mathrm{~Hz}}$. (c) A schematic diagram showing the $\Omega$-exo-endocytosis model, where the fusion-generated $\Omega$-profile may stay at the site (stay fusion), close the pore (close fusion) or shrink until undetectable ( $\Omega$-shrink fusion). For stay and close fusion, the $\Omega$-profile may change in sizes (drawing of different sizes of $\Omega$-profiles). Examples of $\Omega$-shrink (d), stay (e) and close (f) fusion induced by $\operatorname{Train}_{2 \mathrm{~Hz}}: F_{647}$ (red), $F_{488}$ (green), $W_{\mathrm{H}}$ measured from A647 (red) or A488 (green) spot and sampled images (4-15 frame average, A647, red; A488, green, lower) at times indicated (lines) are plotted versus time. Images were collected every 20-40 ms at the confocal cell-bottom setting (A647 excited strongly; A488 excited weakly). $F_{647}$ or $F_{488}$ was normalized to its mean background value before spots appeared. $W_{H}$ was taken before the spot was too dim to measure. The images were obtained at the cell bottom. These settings apply to all similar plots in Figs 1-4. (g) An example showing that $\Omega$-shrink fusion is the majority during $\operatorname{Train}_{2 \mathrm{~Hz}}$ in control: two neighbouring spots (1 and 2) underwent $\Omega$-shrink fusion. Sampled images (lower) were taken at times indicated by triangles below traces (upper). (h,i) Percentages (mean + s.e.m.) of $\Omega$-shrink, stay and close fusion spots induced by $\operatorname{Train}_{2 \mathrm{~Hz}}(n=18$ cells; total spot number: $192 ; \mathbf{h})$ or whole-cell calcium dialysis $(1.5 \mu \mathrm{M}, n=23$ cells, total spot number: $399 ; \mathbf{i})$ in control conditions. 


\section{a}

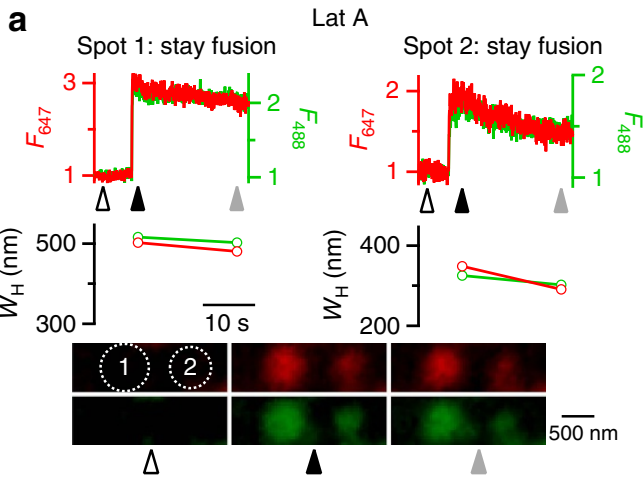

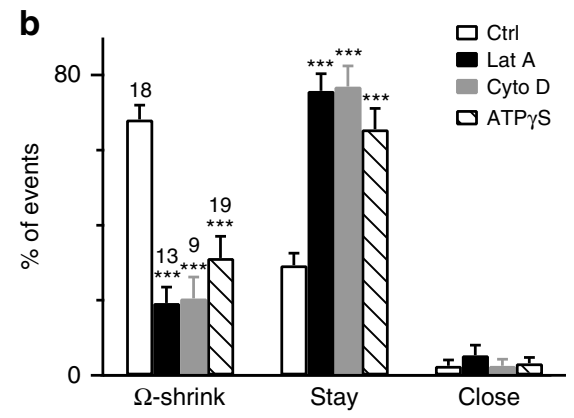

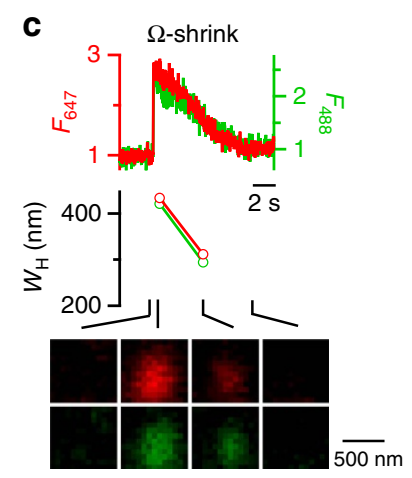

d

e
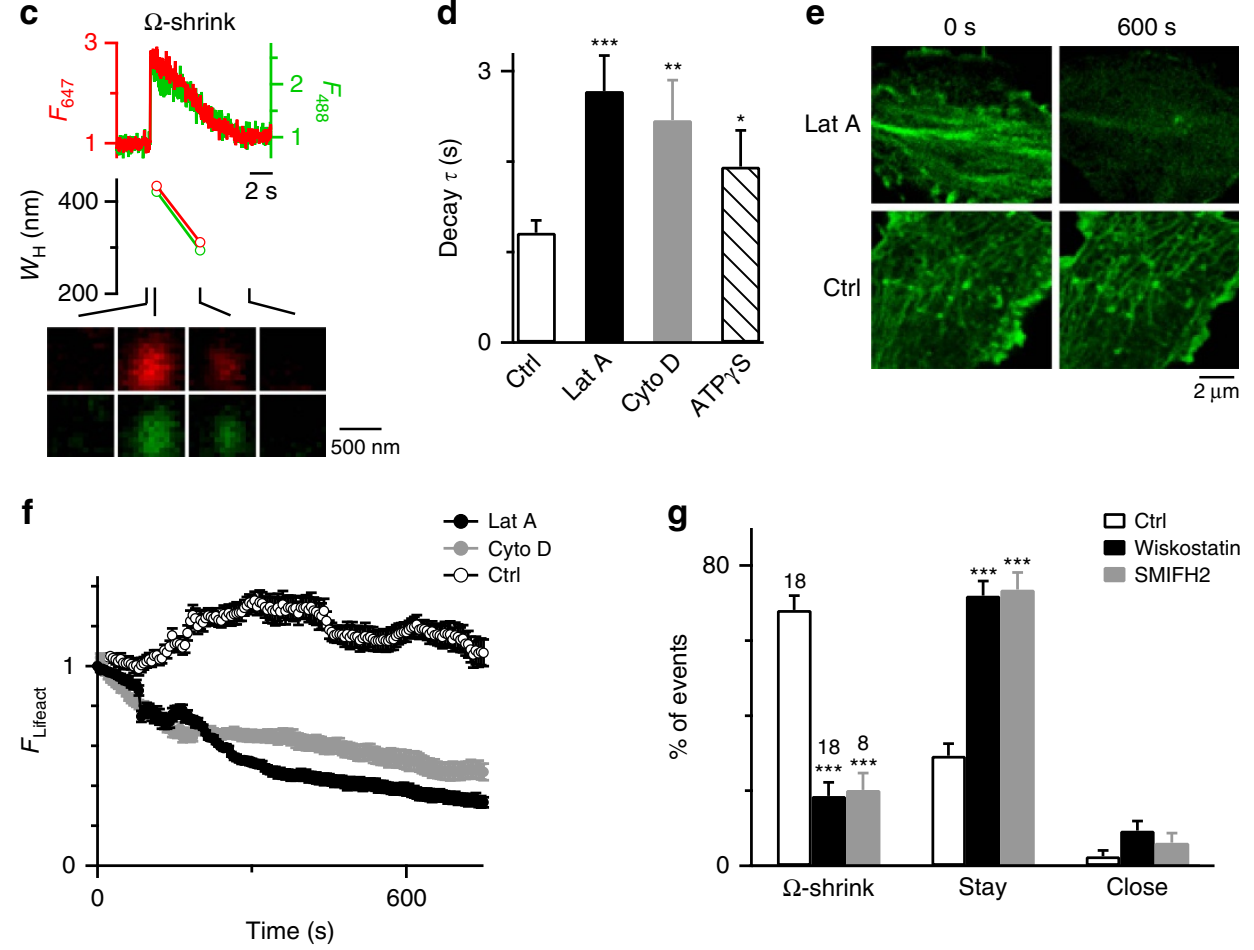

Figure 2 | Block of F-actin inhibits $\boldsymbol{\Omega}$-shrink fusion but promotes stay fusion. (a) An example showing more spots (two neighbouring spots, 1 and 2 ) undergoing stay fusion during $\operatorname{Train}_{2 \mathrm{~Hz}}$ in the presence of Lat $\mathrm{A}(3 \mu \mathrm{M}$, bath). Sampled confocal images were taken at times indicated by triangles.

(b) Percentages (mean + s.e.m.) of $\operatorname{Train}_{2 \mathrm{~Hz}}$-induced spots undergoing $\Omega$-shrink, stay or close fusion in control (Ctrl, same as Fig. $1 \mathrm{~h}, n=18$ cells) or in the presence of $3 \mu \mathrm{M}$ Lat A ( $n=13$ cells; total spot number: 150 ; $\left.{ }^{\star \star \star} P<0.001\right), 4 \mu \mathrm{M}$ Cyto $\mathrm{D}\left(n=9\right.$ cells; 91 spots; $\left.{ }^{\star \star \star} P<0.001\right)$ or ATP $\gamma \mathrm{S}(2 \mathrm{mM}$, replacing $2 \mathrm{mM}$ ATP in the pipette; $n=19$ cells, 213 spots; ${ }^{\star \star \star} P<0.001$ ). Cell numbers are also shown on top of the bar (apples to other similar bar graphs). Error bars indicate s.e.m. (c) A A647/A488-filled spot undergoing $\Omega$-shrink fusion in the presence of Lat A: $F_{647}$ (red), $F_{488}$ (green), $W_{H}$ measured from A647 (red) or A488 (green) spot and sampled images at times indicated (lines) are plotted versus time. Images were obtained at the cell bottom with confocal microscopy. Shrinking rate is slower compared with control in Fig. 1d,g. (d) $F_{647}$ decay $\tau$ (mean + s.e.m.) for $\Omega$-shrink fusion in control ( $n=130$ spots), in the presence of Lat $\mathrm{A}\left(n=33\right.$ spots, $\left.{ }^{\star \star \star} P<0.001\right)$, Cyto $\mathrm{D}\left(n=20\right.$ spots, $\left.{ }^{\star \star} P=0.0016\right)$ or ATP- $\gamma \mathrm{S}\left(n=70\right.$ spots, $\left.{ }^{\star} P=0.0363\right)$. (e) Sampled Lifeact images at the cell bottom before $\left(0 \mathrm{~s}\right.$, left) and $600 \mathrm{~s}$ after (right) application of Lat $\mathrm{A}(3 \mu \mathrm{M})$ or a control (Ctrl) solution. (f) Lifeact-TagGFP2 fluorescence ( $F_{\text {Lifeact, }}$ mean \pm s.e.m.) at the cell bottom before (time 0$)$ and during application of a control solution (Ctrl, 11 cells), Lat A ( $3 \mu \mathrm{M}, 6$ cells) or Cyto D ( $4 \mu \mathrm{M}, 6$ cells). $F_{\text {Lifeact }}$ was normalized to the value at time 0. (g) Percentages (mean + s.e.m.) of $\operatorname{Train}_{2 \mathrm{~Hz}}$-induced spots undergoing $\Omega$-shrink, stay or close fusion in control (Ctrl, same as Fig. 1h) or in the presence of $10 \mu \mathrm{M}$ wiskostatin ( $n=18$ cells; total spot number: $\left.175 ;{ }^{\star \star \star} p<0.001\right)$ or $25 \mu \mathrm{M} \mathrm{SMIFH2} \mathrm{(} n=8$ cells; 74 spots; $\left.{ }^{\star \star \star} P<0.001\right)$ in the bath solution. Statistical test for $\mathbf{b}, \mathbf{d}$ and $\mathbf{g}$ was Student's $t$-test.

Consistent with dynamic F-actin assembly that requires ATP hydrolysis ${ }^{17}$, replacing ATP with adenosine 5-( $\gamma$-thio) triphosphate tetralithium salt (ATP $\gamma \mathrm{S})(2 \mathrm{mM}$, in the pipette solution), a non-hydrolysable ATP analogue, inhibited $\Omega$-shrink fusion and increased stay fusion, but did not affect close fusion (Fig. 2b and Supplementary Fig. 4a). ATP $\gamma \mathrm{S}$ also slowed down the $F_{647}$ decay $\tau$ during $\Omega$-shrink fusion (Fig. $2 \mathrm{~d}$ and Supplementary Fig. 4b). Thus, ATP hydrolysis is needed to merge $\Omega$-profiles.
Although the $\Omega$-shrink fusion frequency is inversely related to $\mathrm{ICa}^{11}$, inhibition of $\Omega$-shrink by actin-related drugs is not due to ICa changes for two reasons. First, these drugs did not affect ICa (Supplementary Fig. 5). Second, Lat A or Cyto D inhibited $\Omega$-shrink and promoted stay fusion induced by whole-cell calcium dialysis, a stimulus independent of ICa (Supplementary Fig. 6).

To strengthen our conclusion, we studied how $\Omega$-profile merging is affected when a major actin isoform, $\beta$-actin (encoded 
by $A c t b$ gene $)^{18}$, is knocked out. KO was achieved by breeding chromaffin cell-specific Cre mice, the PNMT Cre/ + mice ${ }^{19}$, with Actb $^{\text {LoxP/LoxP }}$ mice $^{20}$ and subsequently breeding PNMT ${ }^{\text {Cre/ }+ \text {; }}$ $\mathrm{Actb}^{\mathrm{LoxP} /+}$ mice with $\mathrm{PNMT}^{\mathrm{Cre} /+} ; \mathrm{Actb}^{\mathrm{LoxP} /+}$ mice to generate $\mathrm{PNMT}^{\mathrm{Cre} / \mathrm{Cre}} ; \mathrm{Actb}^{\mathrm{LoxP} / \mathrm{LoxP}}$ mice (Actb ${ }^{-/-}$mice; Supplementary Fig. 7). Immunostaining of Cre and $\beta$-actin confirmed expression of Cre-recombinase and the absence of $\beta$-actin in Actb ${ }^{-1-}$ mouse chromaffin cells (Fig. 3a).

In cultured wild-type (WT) or Actb ${ }^{\text {LoxP/LoxP }}$ mouse chromaffin cells (control), Train ${ }_{2 \mathrm{~Hz}}$ induced an ICa of $416 \pm 55 \mathrm{pA}$ and a capacitance jump ( $n=10$ cells; Supplementary Fig. $8 \mathrm{a}$ ). Fusion events included $\Omega$-shrink fusion ( $80 \pm 5 \%$ ), stay fusion (18 $\pm 5 \%$ ) and close fusion $(2 \pm 1 \%)$ as recorded at the confocal $\mathrm{A} 647_{\text {strong }} /$

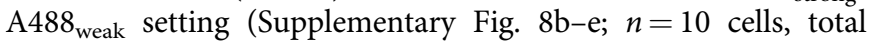
fusion spots: 80 ). Similar percentages of $\Omega$-shrink, stay and close fusion were induced by whole-cell dialysis of calcium $(1.5 \mu \mathrm{M})$ into the chromaffin cells (Fig. 3b). These results were similar to those observed in control bovine chromaffin cells. We used calcium dialysis as stimulation for most mouse experiments, because calcium dialysis induced more fusion events.

$\beta$-Actin $\mathrm{KO}$ reduced $\Omega$-shrink from $77 \%$ in control to $25 \%$ and increased stay fusion from 20 to $74 \%$, but did not affect close fusion during calcium dialysis (Fig. 3b). Similar reduction of $\Omega$-shrink fusion and increase of stay fusion were obtained during Train $_{2 \mathrm{~Hz}}$ in $\mathrm{Actb}^{-1-}$ mouse chromaffin cells (Supplementary Fig. 8e). Rescue of $\mathrm{KO}$ by transfecting WT $\beta$-actin attached with monomeric enhanced green fluorescent protein ( $\beta$-ActinmEGFP, for recognition of transfected cells) to Actb ${ }^{-/}$cells increased $\beta$-actin expression (Fig. $3 c ; n=3$ transfections) and rescued $\Omega$-shrink fusion to the control level (Fig. $3 \mathrm{~b} ; n=7$ cells). Thus, $\beta$-actin is involved in mediating $\Omega$-profile merging.
Application of Lat A $(3 \mu \mathrm{M})$ in $\mathrm{Actb}^{-1-}$ cells did not further reduce $\Omega$-shrink fusion (Fig. $3 \mathrm{~b} ; n=7$ cells), but significantly reduced $\Omega$-shrink fusion in WT cells (Fig. $3 \mathrm{~b} ; n=10$ cells), confirming that Lat A reduces $\Omega$-shrink fusion by blocking F-actin. These results consolidated our conclusion that actin is involved in mediating $\Omega$-shrink fusion.

F-actin is more concentrated near the plasma membrane. Recent studies show that in cells containing $\sim 1-5 \mu \mathrm{m}$ granules, F-actin coats the fusing $\Omega$-profile with an onset at several seconds after fusion, which is thought to either compress or hold the $\Omega$-profile ${ }^{5-10}$. Here we determined whether F-actin coats and shrinks the fusing $\Omega$-profile in chromaffin cells. STED scanning at the microscopic XY plane above the cell bottom $(>2 \mu \mathrm{m})$ revealed that dense Lifeact co-localized with or near the overexpressed phospholipase $\mathrm{C}$ delta $\mathrm{PH}$ domain tagged with mPapaya (PH-mPapaya), which labels PtdIns $(4,5) \mathrm{P}_{2}\left(\mathrm{PIP}_{2}\right)$, a lipid localized at the plasma membrane (Fig. 4a) ${ }^{21}$. STED scanning at the microscopic $X Z$ plane (vertical to $X Y$ plane, parallel to microscopic $Z$ axis) at the cell bottom revealed that Lifeact closely adhered to the PH-mPapaya (Fig. 4b), confirming a dense cortical actin network observed near, but not far from, the plasma membrane (see also Supplementary Fig. 9a,b for quantification $)^{22-24}$. With this dense actin network, granules near the plasma membrane, including NPY-mCherry-containing granules, were more associated with the F-actin network than those far from the plasma membrane (Fig. 4c), similar to a recent report with three-dimensional electron tomography ${ }^{24}$. We did not observe consistently actin rings surrounding NPY-EGFPcontaining vesicles (Fig. 4c).
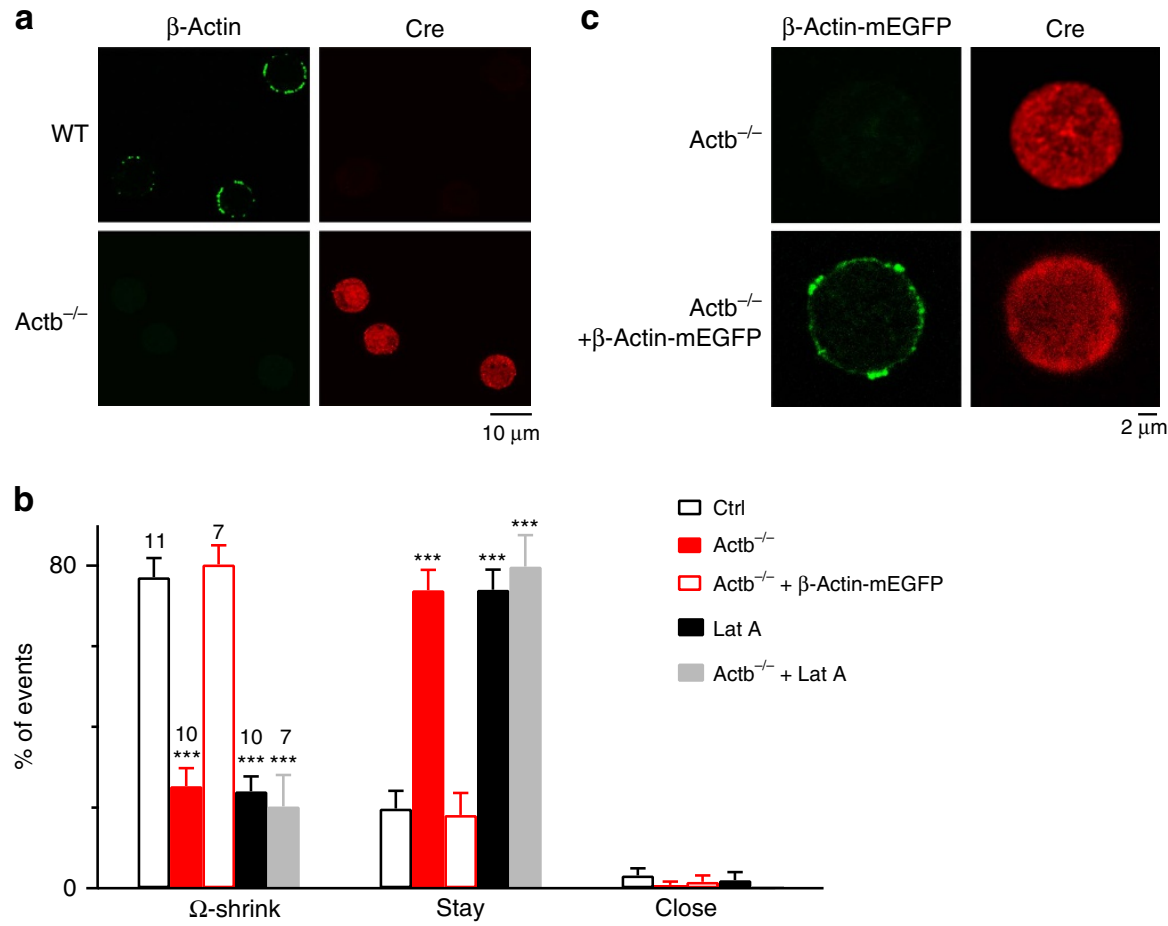

Figure 3 | $\boldsymbol{\beta}$-Actin KO inhibits $\boldsymbol{\Omega}$-shrink fusion but promotes stay fusion. (a) Immunostaining of $\beta$-actin in WT and Actb ${ }^{-/-}$mouse chromaffin cells; Cre antibody staining was also shown to indicate cells expressed with $\mathrm{Cre}$ for deletion of Actb1 gene. (b) Percentages (mean + s.e.m.) of $\Omega$-shrink, stay and close fusion induced by whole-cell calcium $(1.5 \mu \mathrm{M})$ dialysis in control $(n=11$ cells; total spot number: 96$)$, Actb ${ }^{-/-}$cells ( $n=10$ cells; total spot number: 101), Actb ${ }^{-/}$- cells overexpressed with $\beta$-Actin-mEGFP ( $n=7$ cells; total spot number: 59$)$, control cells treated with Lat $A$ ( $n=10$ cells; total spot number: 79 ) and $A c t b^{-/}$cells treated with Lat $A\left(n=7\right.$ cells; total spot number: 63 ). ${ }^{\star \star \star} P<0.001$ (compared with control, Student's $t$-test). (c) $\beta$-Actin-mEGFP in Actb ${ }^{-/}$- cells not transfected (upper) or transfected with $\beta$-Actin-mEGFP (lower). Cre antibody staining was also shown to indicate cells expressed with Cre. 
a $\mathrm{XY}$ scanning (above cell bottom)
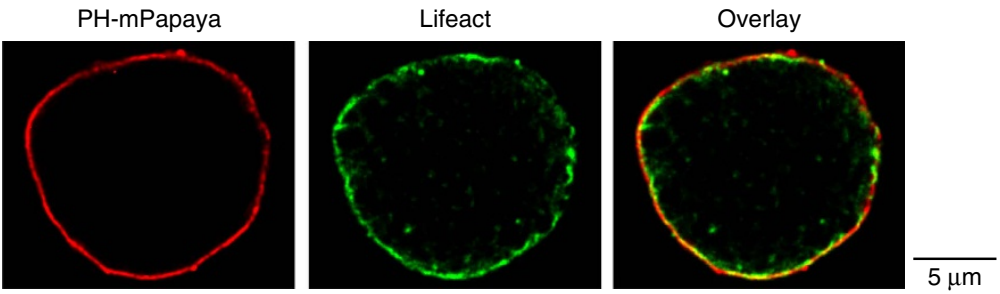

b

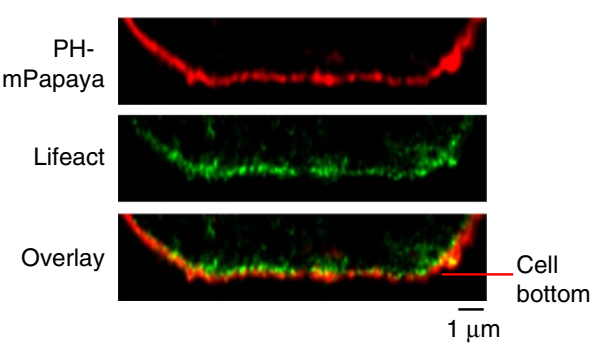

C XZ scanning

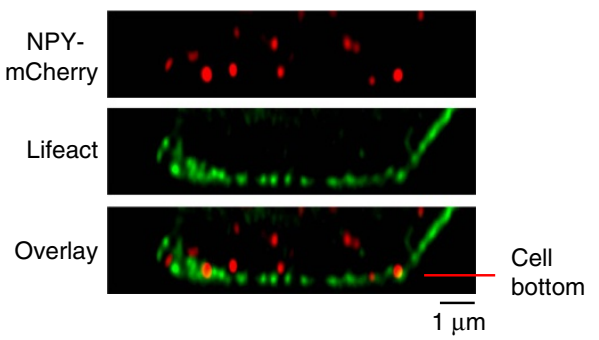

d

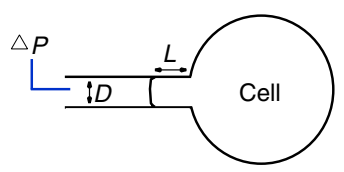

Lat A

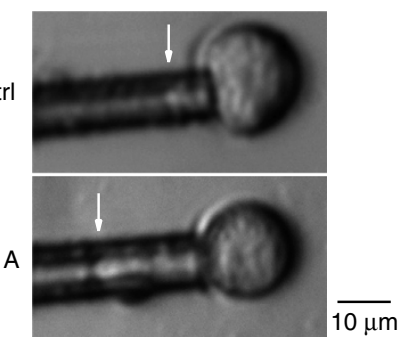

e

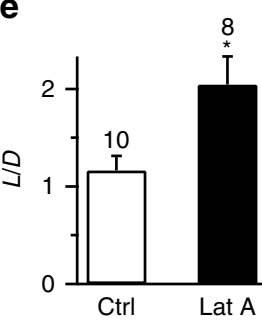

f

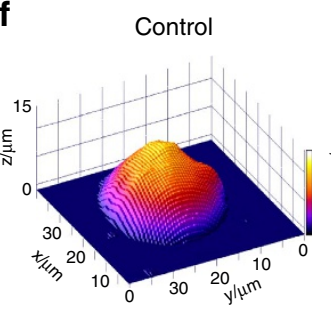

Hyper-osmotic

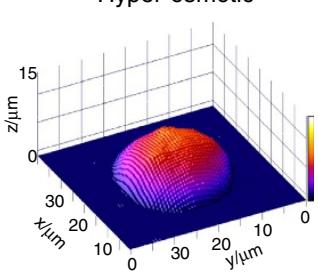

Hypo-osmotic

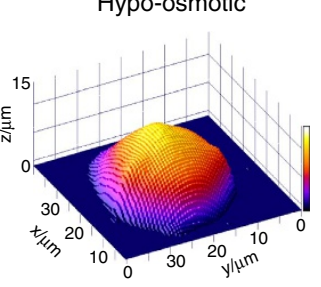

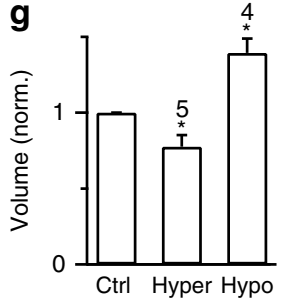

Figure 4 | Localization of F-actin and manipulation of membrane tension by Lat A and by changes of osmolarity. (a) Sampled STED images of a cell overexpressed with PH-mPapaya (left, red, labelling the plasma membrane) and Lifeact-TagGFP2 (middle, green) at the conventional XY scanning mode with a focal plane $>2 \mu \mathrm{m}$ above the cell bottom. Left and middle panels are superimposed in the right panel. (b) STED XZ imaging of PH-mPapaya (upper) and Lifeact-TagGFP2 (middle) at the cell bottom. Upper and middle images are superimposed in the lower panel. (c) Sampled STED images of a cell overexpressing NPY-mCherry (upper, red) and Lifeact (middle, green) at the cell bottom, at the XZ scanning mode. Upper and middle panels are superimposed in the lower panel. (d) Lat $A$ reduces cell surface tension. Left: drawings of micropipette aspiration technique. A negative pressure $(\Delta P)$ on the pipette (with a diameter $D$ ) draws the cell membrane into the pipette by a length $L$. Right: pipette-aspirated cells (bright-field images) in the absence $(C t r l)$ and presence of Lat $A(0.5 \mu \mathrm{M})$. Arrows, membrane projection $(L)$ in the micropipette $(\Delta P=500 \mathrm{~Pa})$. (e) Normalized projection length $(L / D$, mean + s.e.m.) for aspirated cells in the absence (Ctrl, $n=10$ cells) or presence of Lat A $\left(0.5 \mu \mathrm{M}, n=8\right.$ cells; ${ }^{\star} P=0.011$; unpaired two-tailed Student's $t$-test). $\Delta P=500 \mathrm{~Pa}$. (f) Three-dimensional (3D) images of a chromaffin cell in control (Ctrl, $305 \mathrm{mOsm}$ ), in a hyper-osmotic solution (650 mOsm, Hyper) and a hypo-osmotic solution ( $164 \mathrm{mOsm}$, Hypo). The image was obtained with atomic force microscopy. (g) Chromaffin cell volume (mean + s.e.m.) in Ctrl, Hyper ( $n=5$ cells, ${ }^{\star} P=0.03$, paired two-tailed Student's $t$-test) or Hypo solution ( $n=4$ cells, ${ }^{\star} P=0.016$, paired two-tailed Student's $t$-test) computed from atomic force microscopic 3D images. Hyper and Hypo data were normalized to the Ctrl ( $305 \mathrm{mOsm})$.

Confocal imaging revealed that in $41 \%$ (15 out of 37 ) of $\Omega$-shrink fusion events, as $F_{647}$ decayed due to $\Omega$-profile shrinking, $F_{\text {Lifeact }}$ increased at the spot centre (Supplementary Fig. 9c,d), suggesting F-actin movement or coating of the shrinking $\Omega$-profile. However, the onset of $F_{\text {Lifeact }}$ increase was $1.9 \pm 0.4 \mathrm{~s}(n=15)$ later than the onset of $F_{647}$ decay that reflected $\Omega$-profile shrinking (Supplementary Fig. 9c,d). $F_{\text {Lifeact }}$ increase started when $F_{647}$ decreased by $57 \pm 5 \%(n=15$; Supplementary Fig. 9c,d). This delayed $F_{\text {Lifeact }}$ increase suggests that F-actin movement or coating does not initiate $\Omega$-profile shrinking. More importantly, $59 \%$ (22 out of 37 ) of $\Omega$-shrink fusion events were not accompanied with $F_{\text {Lifeact }}$ increase (Supplementary Fig. 9e,f). This lack of $F_{\text {Lifeact }}$ increase was not due to the lack of Lifeact expression, because (1) Lifeact was expressed nearby $\Omega$-shrink events showing no $F_{\text {Lifeact }}$ increase (for example, Supplementary Fig. 9e) and (2) 15 out of $22 \Omega$-shrink events showing no $F_{\text {Lifeact }}$ increase were observed in cells containing other $\Omega$-shrink fusion events with $F_{\text {Lifeact }}$ increase. Thus, $\Omega$-profile shrinking 
takes place regardless of whether F-actin moves into the shrinking $\Omega$-profile or not, suggesting that $\Omega$-profile shrinking does not require F-actin recruitment to the shrinking $\Omega$-profile.

We do not know why F-actin moves into the shrinking $\Omega$-profile in some cases. It could be either a passive refilling of the empty space or an active process. Further understanding of this phenomenon is beyond the scope of the present work, which aimed at understanding the mechanisms underlying $\Omega$-shrink fusion.

F-actin exerts mechanical forces to merge the $\Omega$-profile. F-actin increases the plasma membrane tension $\left(\text { Tension }_{\mathrm{pm}}\right)^{25-28}$. Here we tested whether F-actin shrinks the $\Omega$-profile via an increase of Tension $_{\mathrm{pm}}$. We estimated Tension ${ }_{\mathrm{pm}}$, the force needed to pull the surface by certain length (force per unit length), using the micropipette aspiration technique. A negative pressure applied to a micropipette in contact with the plasma membrane drew the plasma membrane into the pipette to a length $L$ (Fig. $4 \mathrm{~d})^{29}$. The normalized length, calculated as $L$ divided by the pipette diameter $D(L / D)$, is inversely correlated with Young's module, a membrane elasticity measurement that is correlated with Tension $_{\mathrm{pm}}{ }^{29}$. With a negative pressure of $500 \mathrm{~Pa}$, the $L / D$ value was $\sim 2$ times larger in the presence of Lat $\mathrm{A}$ than in control (Fig. 4d,e), suggesting that Lat A reduces membrane stiffness and Tension $_{\mathrm{pm}}$ in chromaffin cells.

To determine whether reduction of Tension $_{\mathrm{pm}}$ inhibits $\Omega$-shrink fusion, we changed Tension ${ }_{\mathrm{pm}}$ with a commonly used method-increasing or reducing the bath solution osmolarity to reduce or increase Tension $_{\mathrm{pm}}$, respectively ${ }^{25,28}$. Consistent with reduced Tension $_{\mathrm{pm}}$, increasing osmolarity from 305 (control) to 650 mOsm (hyper-osmotic solution) reduced the cell volume, measured with atomic force microscopy (AFM; Fig. 4f,g). It also reduced the cell diameter, detected by confocal microscopy at the cell-centre with A647 in the bath (Supplementary Fig. 10a). Consistent with enhanced Tension ${ }_{\mathrm{pm}} 25,28$, reducing the bath osmolarity from 305 to $164 \mathrm{mOsm}$ (hypo-osmotic solution) increased the cell volume (Fig. 4f,g) and diameter (Supplementary Fig. 10b), and increased Tension ${ }_{\mathrm{pm}}$ estimated with micropipette aspiration ( $n=10$ cells, not shown).

Resembling actin blockers, the hyper-osmotic solution decreased $\Omega$-shrink fusion induced by $\operatorname{Train}_{2 \mathrm{~Hz}}$ from 68 to $23 \%$ and increased the stay fusion from 29 to $75 \%(P<0.001$; Fig. $5 \mathrm{a})$. Adding Lat A to a hyper-osmotic solution did not further reduce $\Omega$-shrink or increase the stay fusion percentage (Fig. 5a), suggesting that Lat A inhibited $\Omega$-shrink fusion primarily by reducing Tension pm $_{\text {. }}$

Compared with control, the hypo-osmotic solution did not further increase the $\Omega$-shrink fusion or decrease stay fusion (Fig. 5b), probably because $\Omega$-shrink fusion has already approached its maximal capacity $(\sim 70 \%)$ in control. However, in the presence of Lat $\mathrm{A}$, applying the hypo-osmotic solution increased $\Omega$-shrink fusion from 19 to $50 \%(P<0.001 ; t$-test $)$, decreased stay fusion from 76 to $48 \%(P<0.001$; $t$-test, 18 cells; Fig. 5b) and did not affect ICa (Supplementary Fig. 11). This rescue of $\Omega$-shrink fusion by a large extent (Fig. $5 \mathrm{~b}$ ) suggests that

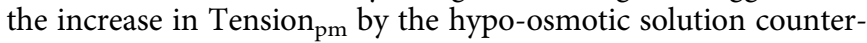
acts Lat A's inhibitory effects on $\Omega$-shrink fusion.

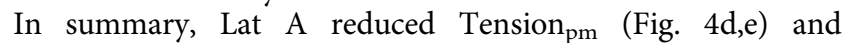
$\Omega$-shrink fusion percentage (Fig. 2b). Reducing Tension pm $_{\text {by }}$ the hyper-osmotic solution mimicked Lat A's effect in inhibiting $\Omega$-shrink fusion and prevented Lat A from further inhibiting $\Omega$-shrink fusion (Fig. 5a). Conversely, enhancing Tension ${ }_{\mathrm{pm}}$ by the hypo-osmotic solution largely prevented Lat A from inhibiting $\Omega$-shrink fusion (Fig. $5 \mathrm{~b}$ ). These results suggest that Lat A inhibits $\Omega$-shrink fusion by reducing Tension $_{\mathrm{pm}}$. In other words, F-actin mediates $\Omega$-shrink fusion by providing sufficient Tension $_{\mathrm{pm}}$.

In Actb $^{-1-}$ cells, applying the hypo-osmotic solution $(221 \mathrm{mOsm})$ increased $\Omega$-shrink fusion from 20 to $78 \%$ $(P<0.001 ; t$-test $)$ and decreased stay fusion from 73 to $20 \%$ $(P<0.001 ; t$-test, $n=10$ cells; Fig. $5 c)$. Applying the hypoosmotic solution to WT mouse chromaffin cells did not increase the $\Omega$-shrink fusion (Fig. $5 \mathrm{c} ; n=8$ cells). Thus, increasing Tension $_{\mathrm{pm}}$ by hypo-osmotic solution rescued the inhibition of $\Omega$-shrink fusion by $\beta$-actin $\mathrm{KO}$, which further supports our suggestion that actin mediates $\Omega$-shrink fusion by providing sufficient Tension $_{\mathrm{pm}}$.

How would sufficient Tension ${ }_{\mathrm{pm}}$ shrink the $\Omega$-profile? Given that blocking F-actin increased stay fusion and thus the number of $\Omega$-profiles at the plasma membrane, there should be an F-actin-independent force that maintains the $\Omega$-profile from collapsing. With this $\Omega$-profile-maintaining force, Tension ${ }_{\mathrm{pm}}$ may pull the $\Omega$-profile membrane and thus shrink the $\Omega$-profile til merging of the $\Omega$-profile with the plasma membrane (Fig. $5 \mathrm{~d}$ ). We can now update the $\Omega$-exo-endocytosis model (Fig. 1c), where calcium and dynamin mediate close fusion ${ }^{11}$, with the involvement of F-actin and Tension ${ }_{\mathrm{pm}}$ in $\Omega$-shrink fusion (Fig. 5e).

F-actin blockers increase $\Omega$-profiles at active zones. To determine whether F-actin mediates $\Omega$-profile merging in synapses, we examined whether block of actin dynamics accumulates $\Omega$-profiles in lamprey giant reticulospinal synapses, in which intermediates of the vesicle cycle can be clearly resolved using $\mathrm{EM}^{30,31}$. We microinjected giant axons with a mixture of Lat $\mathrm{A}$ and Cyto D or a modified profilin-actin protein with a zerolength covalent cross-link between profilin and actin, termed $\mathrm{PxA}^{32-34}$. PxA structually resembles endogeneous profilin-actin, but is non-dissociable between profilin and actin, and thus inhibits formin-dependent F-actin assembly ${ }^{32-34}$. Concentrations of the actin-directed compounds in microinjection pipette were $60 \mu \mathrm{M}$ per $33 \mu \mathrm{M}$ for Lat A/Cyto D and $5 \mathrm{mg} \mathrm{ml}^{-1}$ for PxA, but were diluted $\sim 10-20$ times in the studied regions of axons following microinjections (Supplementary Fig. 12a). The active zone morphology (Supplementary Fig. 12b-e and Fig. 6a,b) and the action potential progagation along the axon (Supplementary Fig. 12f) were apparently normal after microinjection. Synapses were stimulated with action potentials at $5 \mathrm{~Hz}$ for $20-30 \mathrm{~min}$, then fixed and embedded for EM in serial ultrathin sections 30,31

Vesicle clusters were observed at active zones and examined in serial ultrathin sections (Fig. 6a-f and Supplementary Fig. 12b-d). $\Omega$-profiles were identified as having a pore smaller than or equal to its width (Fig. 6c-e). $\Omega$-profiles were rarely observed in control synapses or synapses microinjected with bovine profilin I (Fig. 6c,g), but were much more frequently observed at the active zones of the synapses microinjected with Lat A/Cyto D (Fig. 6d,g) or PxA (Fig. 6e,g). Lat A/Cyto D or PxA did not significantly affect the number of vesicles at the active zone membrane (Supplementary Fig. 12b-e). Unlike clathrin-coated $\Omega$-profiles observed at the periactive zone (Fig. $6 \mathrm{f})^{30,31}, \Omega$-profiles at the active zone were not coated with the clathrin lattice (Fig. 6c-e), suggesting that they are not generated via clathrinmediated endocytosis. Dimension of most of $\Omega$-profiles were similar to vesicles, but some appeared to be larger or smaller (for example, Fig. 6d,e and Supplementary Fig. 12b-d), consistent with stay fusion displaying variations of $\Omega$-profile size in chromafin cells (Supplementary Fig. 1) ${ }^{11}$.

The $\Omega$-profiles accumulated at the active zone are unlikely to be due to block of the fusion pore closure, because in chromaffin cells actin blockers did not block close fusion induced by $\operatorname{Train}_{2 \mathrm{~Hz}}$ (Fig. 2) or a $1 \mathrm{~s}$ depolarization ( $n=8$ cells, $3 \mu \mathrm{M}$ Lat $\mathrm{A}$, not 
a

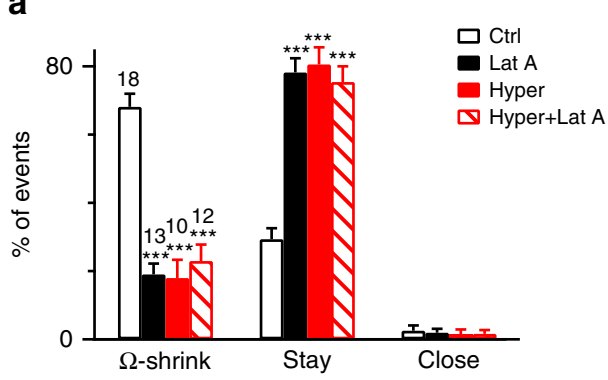

C

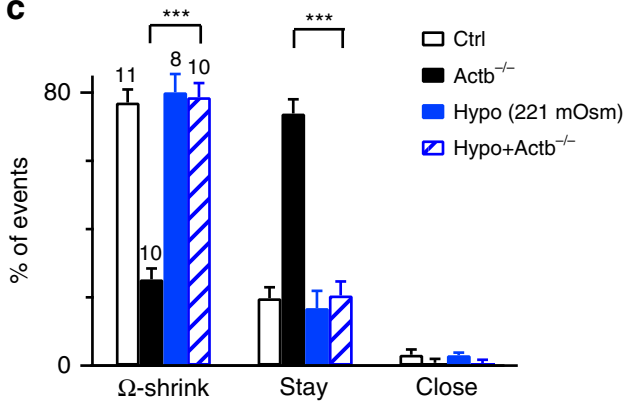

e

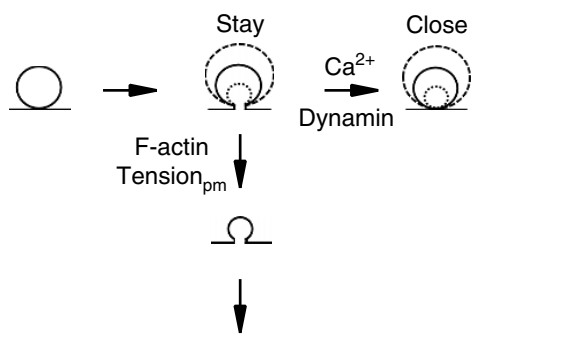

b

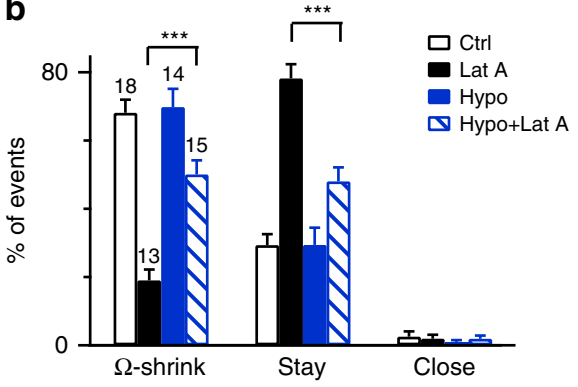

d

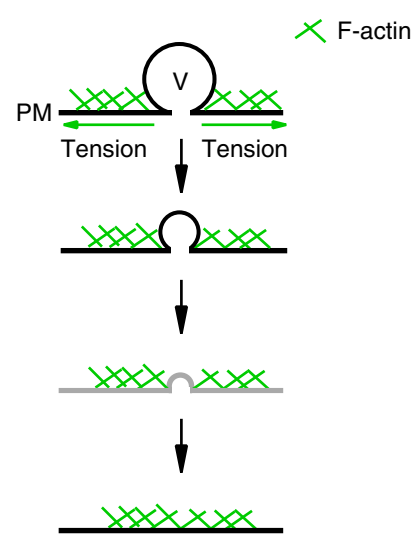

$\Omega$-shrink fusion

Figure 5 | Actin provides sufficient Tension ${ }_{\mathbf{p m}}$ to mediate $\boldsymbol{\Omega}$-profile shrinking. (a) Percentages (mean + s.e.m.) of $\Omega$-shrink, stay and close fusion induced by $\operatorname{Train}_{2 \mathrm{~Hz}}$ in $\mathrm{Ctrl}$ ( $305 \mathrm{mOsm}, n=18$ cells; 192 spots in total), in the presence of Lat A ( $3 \mu \mathrm{M}, 305 \mathrm{mOsm}, n=13$ cells; 150 spots; $\left.{ }^{\star \star \star} P<0.001\right)$, in Hyper solution ( $n=10$ cells; 70 spots, $\left.{ }^{\star \star \star} P<0.001\right)$ and in Hyper solution containing Lat $A\left(n=12\right.$ cells; 100 spots, $\left.{ }^{\star \star \star} P<0.001\right)$ in bovine chromaffin cells. Statistical significances were assessed by unpaired two-tailed Student's $t$-test (applies to a-c). (b) Percentages (mean + s.e.m.) of $\Omega$-shrink, stay and close fusion induced by $\operatorname{Train}_{2 \mathrm{~Hz}}$ in Ctrl (305 mOsm, 18 cells, 192 spots), in the presence of Lat A ( 305 mOsm), in Hypo solution ( $n=14$ cells, 220 spots; $P=0.665)$ and in Hypo solution containing Lat $A\left(n=15\right.$ cells, 276 spots, $\left.{ }^{\star \star \star} P<0.001\right)$ in bovine chromaffin cells. (c) Percentages (mean + s.e.m.) of $\Omega$-shrink, stay and close fusion induced by whole-cell calcium $(1.5 \mu \mathrm{M})$ dialysis in WT mouse chromaffin cells (WT, $305 \mathrm{mOsm}, 11$ cells, 96 spots), in Actb ${ }^{-/}$- cells $(305 \mathrm{mOsm}, n=10$ cells, 101 spots), in hypo-osmotic solution ( $221 \mathrm{mOsm})$ in WT cells $(n=8$ cells, 60 spots) and in the hypo-osmotic solution in Actb ${ }^{-/}$mouse chromaffin cells $\left(n=10\right.$ cells, 82 spots, $\left.{ }^{\star \star \star} P<0.001\right)$. (d) A model showing that F-actin shrinks the $\Omega$-profile by enhancing tension ( $\mathrm{T}$ ) at the plasma membrane (PM). Drawings in black are supported by our data. Drawings in grey are hypothetical (below our resolution limit). As the $\Omega$-profile height equals its pore size, $\Omega$-profile shrinking becomes $\Omega$-profile collapse. (e) Updated $\Omega$-exo-endocytosis model where F-actin provides Tension $_{\mathrm{pm}}$ to mediate $\Omega$-shrink fusion and calcium triggers a dynamin-dependent close fusion (taken from ref. 11).

shown), the latter of which induced more close fusion events ${ }^{11}$. Analogous to results observed in chromaffin cells, results observed in lamprey axons (Fig. 6) suggest that F-actin promotes $\Omega$-profile merging at active zones. This suggestion was further supported by the following two sets of control experiments.

First, post-embedding immunoEM with a monoclonal actin antibody $^{12,19}$ revealed an accumulation of immunogold particles around dense projections of the active zone in giant synapses (Fig. 6h-j and Supplementary Fig. 13a-d) ${ }^{35}$. The number of gold particles near the presynaptic plasma membrane $(<100 \mathrm{~nm})$ of the active zone $\left(95.7 \pm 15.4 \mu \mathrm{m}^{-2} ; n=20\right.$ synapses) was significantly higher than that (the particles near the presynaptic plasma membrane) $>300 \mathrm{~nm}$ outside the active zone (PM outside active zone, $16.9 \pm 5.0 \mu \mathrm{m}^{-2} ; \quad n=20$ synapses, $P<0.001$; Fig. $6 \mathrm{j}$ and also see Supplementary Fig. 13a-d for illustrations). It was also significantly higher than in the synaptic vesicle reserve pool area, $200 \mathrm{~nm}$ inside the active zone membrane $\left(43.5 \pm 5.0 \mu \mathrm{m}^{-2} ; n=17\right.$ synapses, $P=0.0048$; Fig. $\left.6 \mathrm{~h}-\mathrm{j}\right)$. Thus, actin network is denser near the active zone membrane. The dense actin network at the active zone membrane was further confirmed by confocal microscopy of F-actin labelled with phalloidin conjugated with Alexa Flour 488 (phalloidin-Alexa 488) injected into the axon (Supplementary Fig. 14a). Microinjected phalloidin-Alexa 488 accumulated in spots of fluorescence that labelled active and periactive zones ${ }^{36}$ (see also Supplementary Fig. 14a).

Second, to determine whether Lat $\mathrm{A}$ and Cyto $\mathrm{D}$ reduced F-actin, we injected Lat A/Cyto D plus Texas Red and 10-15 min later injected phalloidin-Alexa 488 into the axon at $\sim 800 \mu \mathrm{m}$ away. Next, we measured phalloidin-Alexa 488 fluorescence punta at $\sim 300-500 \mu \mathrm{m}$ away from the Lat A/Cyto D injection site (Supplementary Fig. 14b,c). The number of detectable puncta 
a

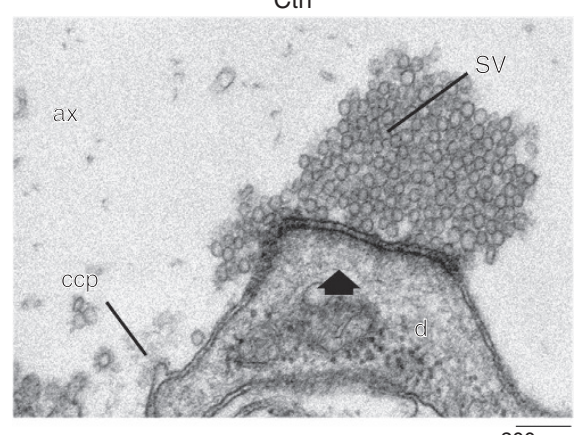

$2 \overline{\mathrm{nm}}$

d
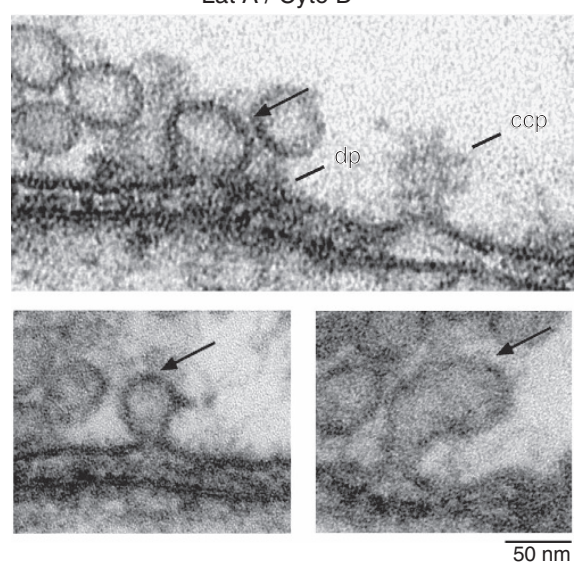

h

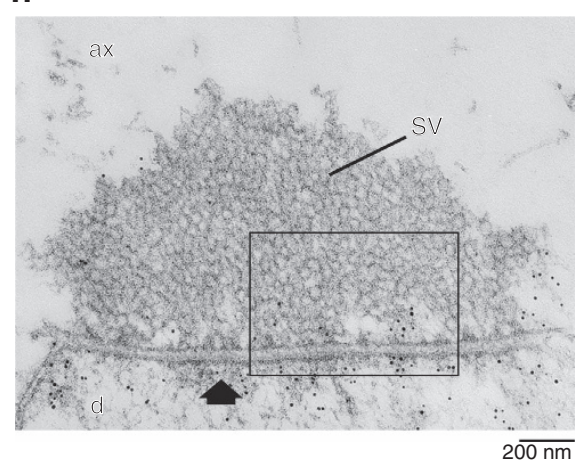

b

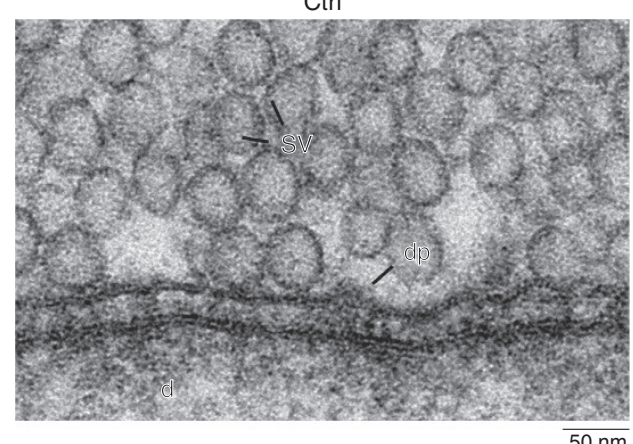

e

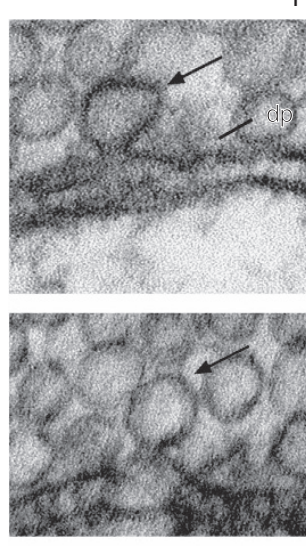

i

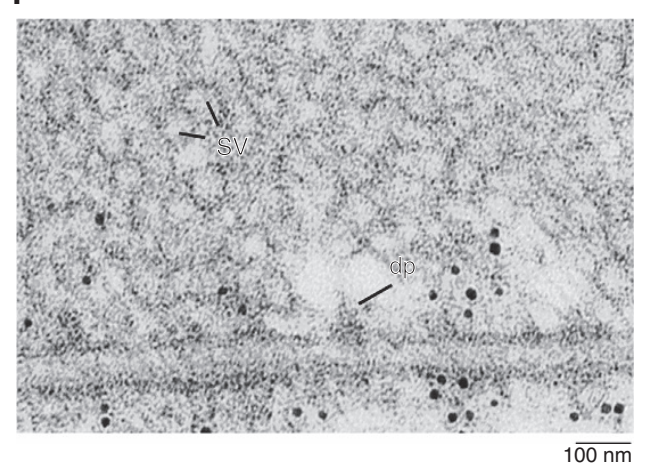

C

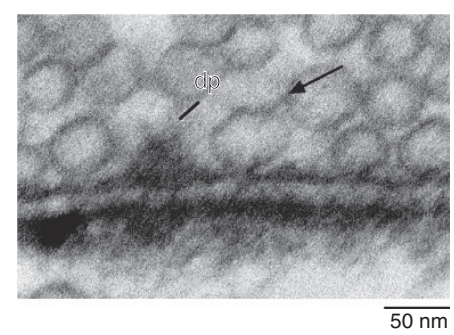

f

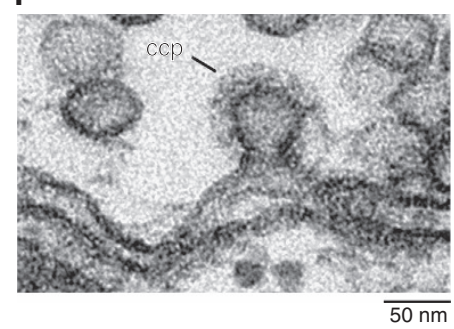

g

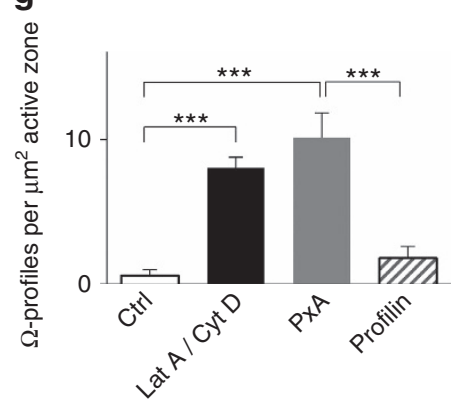

j

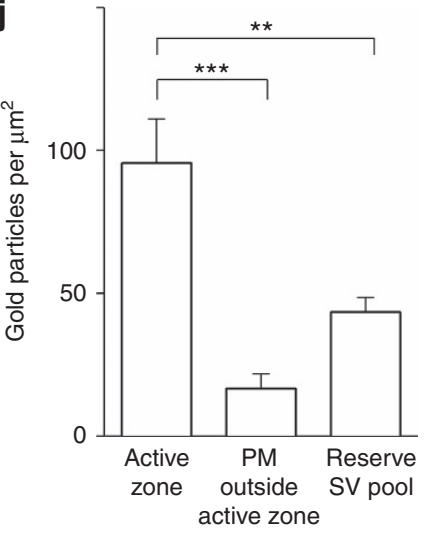

Figure 6 | Actin-directed compounds induce $\boldsymbol{\Omega}$-profiles at lamprey active zones. (a) Electron micrograph of an active zone with a vesicle cluster (arrow) in a control reticulospinal synapse. The axon was stimulated at $5 \mathrm{~Hz}$ for $30 \mathrm{~min}$. A clathrin-coated pit (ccp) is present lateral to the active zone. ax, axoplasmic matrix; d, dendrite; sv, synaptic vesicle. (b) An area of the active zone from a shown at higher magnification. dp, dense projection of the active zone. (c) An EM image showing a rare observation of an $\Omega$-profile at the active zone membrane in the control active zone stimulated at $5 \mathrm{~Hz}$ for $30 \mathrm{~min}$. $(\mathbf{d}, \mathbf{e})$ EM images of active zones in synapses microinjected with Lat A and Cyto $D(\mathbf{d})$ or with PxA (e) and stimulated at $5 \mathrm{~Hz}$ for 30 min. $\Omega$-profiles are marked with thin arrows. (f) An EM image showing clathrin-coated pits at the lateral region away from the active zone of a PxA microinjected synapse. (g) The number of $\Omega$-profiles (mean + s.e.m.) at active zones in control (Ctrl) axons and in axons microinjected with Lat A ( $60 \mu M)$ and Cyto D ( $33 \mu M$ ), with PXA ( $\left.5 \mathrm{mg} \mathrm{ml}^{-1}\right)$ or with profilin I $\left(5 \mathrm{mg} \mathrm{ml}^{-1}\right)\left({ }^{\star \star \star} P<0.001\right)$. Data are plotted as mean + s.e.m. per $\mu \mathrm{m}^{2}$ of active zone (AZ). Each group was from five synapses cut in serial section from three axons. Statistical significances were tested with one-way analysis of variance (ANOVA), $\alpha$-level of 0.05 , followed by the Holm-Šídák's test. ${ }^{\star \star \star} P<0.001$ for Ctrl versus Lat $A / C y t o ~ D, C t r l$ versus PxA or Profilin versus PxA. $P=0.43$ for Ctrl versus Profilin. (h) Immunogold localization of actin immunoreactivity at active zones. A synapse fixed at rest showing gold particles near the presynaptic active zone and the postsynaptic density. (i) The box region in $\mathbf{h}$ shown at higher magnification. (j) Bar graph showing the number of actin gold particles per $\mu \mathrm{m}^{2}$ within $100 \mathrm{~nm}$ from the active zone membrane, within $100 \mathrm{~nm}$ from the presynaptic membrane, but $\sim 300 \mathrm{~nm}$ away from the active zone (PM outside active zone, see Supplementary Fig. 13a for an illustration of these regions), and in the reserve pool area $200 \mathrm{~nm}$ from the active zone ( $n=20$ synapses, mean + s.e.m.; ${ }^{\star \star} P<0.01 ;{ }^{\star \star \star} P<0.001$, Student's $t$-test $)$. 
was decreased by five- to tenfold in the area where compounds intermixed and the average phalloidin-Alexa 488 punta intenstiy was significantly lower in Lat A/Cyto D-injected axons (43.1 \pm 5.1 arbitrary units, $n=15$ spots, 2 axons; Supplementary Fig. 14c,d) than in control axons injected with Texas Red only $(72.8 \pm 2.0$ arbitrary units, $n=29$ spots, 2 axons; Supplementary Fig. 14b,d; $P<0.0001$, two-tailed Student's $t$-test), suggesting that Lat A and Cyto D reduced F-actin in lamprey axons.

In summary, actin was localized in the active zone membrane (Fig. $6 \mathrm{~h}-\mathrm{j}$ and Supplementary Figs 13 and 14). F-actin directed compounds reduced F-actin (Supplementary Fig. 14) in the axon and caused accumulation of $\Omega$-profiles at the active zone membrane (Fig. 6) without damaging the morphology and the action potential propagation of the axon (Supplementary Figs 12 and 13). These results suggest that F-actin is involved in mediating $\Omega$-profile merging at the neuronal nerve terminal.

\section{Discussion}

The present work establishes a molecular and biophysical model accounting for $\Omega$-profile merging, which defines the modes of exocytosis and endocytosis. We found that dynamic F-actin assembly is necessary for $\Omega$-profile merging in chromaffin cells (Figs 1-3). F-actin imaging and Tension $_{\mathrm{pm}}$ measurements and manipulations suggest that F-actin provides sufficient Tension $\mathrm{pm}_{\mathrm{pm}}$ to facilitate $\Omega$-profile shrinking and thus to merge the $\Omega$-profile with the plasma membrane (Figs 4 and 5). Not only F-actin but also the F-actin assembly pathway such as N-WASP, formin (Figs 2 and 6) and ATP hydrolysis (Fig. 2) may participate in $\Omega$-profile merging. The highly abundant cytoskeletal actin and its assembly pathway, the essential energy-providing molecule ATP and cell membrane tension controlled by many factors may thus regulate exo- and endocytosis in physiological and pathological conditions by controlling $\Omega$-profile merging.

Our findings, obtained from bovine and mouse chromaffin cells containing large vesicles, may apply to many other cells containing similarly large dense-core vesicles. They may apply to large vesicles distributed widely in glial cells, nerve terminals, neuronal dendrites and cell bodies that secrete dopamine, peptides or hormones (for example, oxytocin and vasopressin) or deliver transmembrane receptors to dendrites and dendritic spines crucial for our brain's physiological functions and disorders ${ }^{2,37}$.

Our finding that F-actin mediates $\Omega$-profile merging is likely to apply to small synaptic vesicles, because (1) actin blockers accumulate $\Omega$-profiles at lamprey active zones (Fig. 6) and (2) neuroendocrine chromaffin cells and nerve terminals are similar in the molecular machineries of exo- and endocytosis ${ }^{1,2,38-40}$. We acknowledge that the evidence obtained with EM at lamprey synapses was not as direct as that in live chromaffin cells where $\Omega$-profile merging could be observed directly. The final resolution at live synapses, although technically not available now, will eventually be needed to establish our conclusion for synapses.

$\Omega$-profile merging is via shrinking of $\Omega$-profile in chromaffin cells $^{11}$, but is generally believed to be via pore dilation (full-collapse fusion) at synapses ${ }^{41-43}$. One may wonder how F-actin mediates these two different behaviours. $\Omega$-shrink fusion was revealed in live cells with STED imaging at $\sim 90 \mathrm{~nm}$ resolution, which could not exclude pore dilation below $90 \mathrm{~nm}^{11}$, whereas pore dilation was suggested based on $\mathrm{EM}^{41-43}$ but has not been visualized and proved in any live cell. $\Omega$-profile shrinking and pore dilation may therefore be reconciled with a scenario, in which $\Omega$-profile membrane was pulled into the plasma membrane by Tension $_{\mathrm{pm}}$, resulting in continuous $\Omega$-profile shrinking until the height of the $\Omega$-profile is shorter than the pore diameter (Fig. 5d, grey drawing). Under this condition, further pulling of the $\Omega$-profile membrane into the plasma membrane by Tension $_{\mathrm{pm}}$ results in a collapse-like structure, turning into a full-collapse fusion (Fig. 5d). This model requires an actin-independent mechanism to hold the pore from collapse, which must exist because F-actin inhibition increased stay fusion, the persistent presence of fusion-generated $\Omega$-profiles (Figs 2 and 3 ). The pore size may determine when the merging process changes from shrinking to collapse. It would be of great interest to test this model by visualizing the fusion pore during shrinking of the $\Omega$-profile in the future.

Although inhibition of F-actin reduced $\Omega$-shrink, but increased stay fusion, close fusion did not increase (Figs 2 and 3). This observation suggests that conversion from stay to $\Omega$-profile closure is not automatic but requires a trigger. We recently found that calcium influx triggered close fusion-large calcium influx during a $1 \mathrm{~s}$ depolarization induce close fusion in $>80 \%$ of fusion events and nearly no $\Omega$-shrink fusion ${ }^{11}$. Thus, large calcium influx is needed to convert stay to close fusion. This may explain why with relatively low calcium influx during $\operatorname{Train}_{2 \mathrm{~Hz}}$, increase of stay fusion in the presence of F-actin blockers did not significantly increase close fusion. Taken together, the extent of calcium influx determines whether the fusing $\Omega$-profile closes or not (Fig. 5e). If the $\Omega$-profile does not close, sufficient Tension pm provided by F-actin may mediate $\Omega$-shrink fusion (Fig. 5e). If Tension $_{\mathrm{pm}}$ is insufficient, $\Omega$-profile stays at the plasma membrane, resulting in the stay fusion (Fig. 5e).

With large calcium influx, close fusion occurs much more often than $\Omega$-shrink fusion ${ }^{11}$. This might be in part due to inhibition of $\Omega$-shrink fusion by strong calcium influx. Consistent with this possibility, calcium influx facilitates F-actin disassembly ${ }^{44}$, which may result in reduction of $\Omega$-shrink fusion (Fig. 2). It would be of great interest to prove (or disapprove) this possibility with experimental evidence in the future.

A large number of previous studies suggest that actin may play several possibly overlapping roles in the fusion process, including serving as a fusion barrier or providing a scaffold for anchoring secretory vesicles, a track to deliver secretory vesicles to the fusion site, regulation of vesicle docking and priming, regulation of the fusion pore, regulation of membrane merging after fusion and endocytosis (reviewed in ref. 45). As discussed below, the present work suggests a new mechanism of F-actin-mediating $\Omega$-profile shrinking and merging by providing sufficient Tension $\mathrm{pm}_{\mathrm{pm}}$.

In Xenopus oocytes containing cortical granules ${ }^{5}$, human endothelial cells containing Weibel-Palade bodies, lacrimal epithelial acinar cells containing zymogen granules ${ }^{6}$, parotid or pancreatic acinar cells containing zymogen granules ${ }^{7,8}$ and type II pneumocytes containing lamellar body ${ }^{9}$, vesicle fusion is followed by F-actin coating of the fusing vesicle (reviewed in ref. 10). Such an F-actin coating has been suggested to mediate two seemingly opposite functions: to compress the fusing $\Omega$-profile and thus to expel the vesicular content, or to hold the $\Omega$-profile from merging into the plasma membrane. However, these results were obtained from vesicles $(\sim 1-5 \mu \mathrm{m}$ in diameter), which are much larger than those in neuroendocrine chromaffin cells $(\sim 300 \mathrm{~nm})$ and neurons $(\sim 20-80 \mathrm{~nm})$, and take much longer time, in the order of tens of seconds to tens of minutes, to merge the $\Omega$-profile and expel vesicular contents. These substantial differences might explain why F-actin recruitment to the fusing vesicle is not the main mechanism to shrink the $\Omega$-profile in chromaffin cells (Supplementary Fig. 9). Instead, F-actin provides sufficient Tension $_{\mathrm{pm}}$ (Figs 4 and 5) to mediate rapid $\Omega$-profile merging in hundreds of milliseconds in chromaffin cells (Figs 2 and 3). It is possible that this mechanism may also contribute to mediate $\Omega$-profile merging in cells containing large $1-5 \mu \mathrm{m}$ vesicles, given

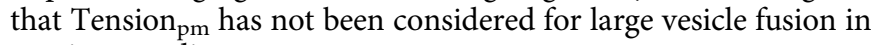
previous studies. 
F-actin blockers slow down catecholamine release ${ }^{46-48}$ and NPY-EGFP release (Supplementary Fig. 15) in chromaffin cells, suggesting that $\mathrm{F}$-actin speeds up vesicular content release ${ }^{49}$. It was suggested that F-actin may squeeze the fusing $\Omega$-profile to speed up vesicular content release ${ }^{49}$. Our results suggest that the force to expel vesicular contents may be from Tension $_{\mathrm{pm}}$ provided by F-actin, but not from active F-actin coating of the fusing $\Omega$-profile as observed during extremely slow release from very large vesicles ${ }^{10}$. In addition to facilitating content release, F-actin may promote dispersion of vesicular membrane proteins by promoting $\Omega$-profile shrinking, because a stabilized $\Omega$-profile retains vesicular membrane protein VAMP2 (ref. 11).

A previous study observed that actin-directed compounds increase the quantal size (from amperometric measurements) and reduce the capacitance variance ${ }^{48,50}$ in chromaffin cells. This observation led to the suggestion that actin stabilizes $\Omega$-profiles in chromaffin cells containing $\sim 300 \mathrm{~nm}$ vesicles ${ }^{50}$. However, this suggestion is interpreted from the measurements of quantal size (from amperometric measurements) and capacitance variance, which could not estimate a pore beyond $\sim 5 \mathrm{~nm}^{51,52}$ nor predict $\Omega$-profile merging. By direct measurements of $\Omega$-profile merging, we found that F-actin mediates $\Omega$-profile merging. We therefore suggest not to interpret $\Omega$-profile merging based on indirect amperometric or capacitance measurements.

Although fusion pore opening is well known to overcome a significant energy barrier ${ }^{4}$, once the pore is opened, the $\Omega$-profile is generally considered an unstable fusion intermediate that rapidly and passively merges with the plasma membrane without consuming energy in endocrine cells and neurons. This view appears to be incorrect, as we found that ATP hydrolysis and dynamic actin polymerization are needed to merge the $\Omega$-profile. The fusion-generated $\Omega$-profile is actually a stable structure in the absence of ATP hydrolysis (Fig. 2). Given that $\Omega$-profile merging takes place in all secretory cells ${ }^{1,2}, \Omega$-profile merging might be an energy-consuming step previously unrecognized. As vesicle exo- and endocytosis consume most ATP molecules in nerve terminals ${ }^{53}$, ATP consumption in $\Omega$-profile merging is a factor worth of consideration in low metabolic states with low ATP levels, mitochondria dysfunctions in various diseases (for example, Parkinson's disease and Alzheimer's disease) that reduce $\mathrm{ATP}^{54}$ and in diseases that affect $\mathrm{F}$-actin or F-actin assembly pathways ${ }^{55}$.

\section{Methods}

Primary bovine chromaffin cell culture. Bovine chromaffin cells were prepared as described previously ${ }^{11}$. Briefly, fresh adrenal glands were obtained from a local slaughterhouse on the day of culture. After excess fat was trimmed off the glands (two were used per culture), glands were then perfused with cold $1 \times$ Lock's buffer containing (in $\mathrm{mM}$ ): $\mathrm{NaCl}, 145 ; \mathrm{KCl}, 5.4 ; \mathrm{Na}_{2} \mathrm{HPO}_{4}, 2.2 ; \mathrm{NaH}_{2} \mathrm{PO}_{4}, 0.9$; glucose, 5.6; and HEPES, $10 \mathrm{pH} 7.3$, to remove any residual blood. Each gland was then injected through the portal vein with $\sim 2 \mathrm{ml}$ of filtered Lock's buffer containing collagenase $\mathrm{P}\left(1.5 \mathrm{mg} \mathrm{ml}^{-1}\right.$, Roche), trypsin inhibitor $\left(0.325 \mathrm{mg} \mathrm{ml}^{-1}\right.$, Sigma) and BSA ( $5 \mathrm{mg} \mathrm{ml}^{-1}$, Sigma), and incubated at $37^{\circ} \mathrm{C}$ for $20 \mathrm{~min}$ in a water bath. The glands were then cut open longitudinally, to expose the digested medulla. The medulla were carefully removed, minced in Lock's buffer and filtered through a nylon mesh. The filtrate was centrifuged at 500 r.p.m. for $4-5 \mathrm{~min}$, to obtain the cell pellet. The supernatant was then removed and the pellets resuspended in Lock's buffer. The process was repeated one to two times until the supernatant was clear. Final cell pellet was resuspended in pre-warmed DMEM low glucose medium (Gibco) supplemented with $10 \%$ fetal bovine serum (Gibco) and plated onto poly-D-lysine and laminin I-coated $25 \mathrm{~mm}$-diameter glass coverslips (Neuvitro Corp., USA). The plated cells were incubated at $37^{\circ} \mathrm{C}, 8 \%$ humidified $\mathrm{CO}_{2}$ and used within 4 days after culturing. In some experiments, cells were transfected with $2 \mu \mathrm{g}$ of Lifeact-tagGFP2 (Ibidi, Germany) by electroporation using a basic neuron nucleofector kit (Lonza, Program O-005) according to the manufacturer's instruction.

Electrophysiology. Whole-cell voltage-clamp and capacitance recordings were performed with an EPC-10 amplifier together with the lock-in software (PULSE, HEKA, Lambrecht, Germany) ${ }^{56}$. All experiments were carried out at room temperature $\left(21^{\circ} \mathrm{C}-24^{\circ} \mathrm{C}\right)$ with the cells immersed in bathing solution containing (in mM): $125 \mathrm{NaCl}, 10$ glucose, $10 \mathrm{HEPES}, 5 \mathrm{CaCl}_{2}, 1 \mathrm{MgCl}_{2}, 4.5 \mathrm{KCl}$ 0.001 tetrodotoxin and 20 tetraethylammonium ion, $\mathrm{pH}$ 7.3. The osmolarity of the bath solution was 305-310 mOsm. In some experiments, we increased the osmolarity to $640-650 \mathrm{mOsm}$ by adding $295 \mathrm{mM}$ sucrose to the bath solution. In some experiments, we decreased the osmolarity to $160-221$ mOsm by reducing $\mathrm{NaCl}$ to $55-70 \mathrm{mM}$.

The pipette (3-6 M $\Omega$ ) solution contained (in $\mathrm{mM}$ ): 130 glutamate, 0.5 EGTA, $12 \mathrm{NaCl}, 30 \mathrm{HEPES}, 1 \mathrm{MgCl}_{2}, 2 \mathrm{ATP}$ and $0.5 \mathrm{GTP}$ pH 7.2 adjusted with $\mathrm{CsOH}$. The osmolarity was $\sim 308 \mathrm{mOsm}$. In some experiments, ATP $(2 \mathrm{mM})$ in the pipette solution was replaced with a non-hydrolysable ATP $\gamma \mathrm{S}\left(2 \mathrm{mM}\right.$, Sigma) and $\mathrm{MgCl}_{2}$ was raised to $3 \mathrm{mM}$ to facilitate endogenous ATP turnover ${ }^{57}$. For intracellular $\mathrm{Ca}^{2}+$ dialysis experiments, the pipette solution contained (in $\mathrm{mM}$ ): 110 glutamate, 10 EGTA, $12 \mathrm{NaCl}, 30 \mathrm{HEPES}, 1 \mathrm{MgCl}_{2}, 2$ ATP $0.5 \mathrm{GTP}$ and $9 \mathrm{CaCl}_{2} \mathrm{pH} 7.2$ adjusted with $\mathrm{CsOH}$. The free $\mathrm{Ca}^{2}+$ concentration was $\sim 1.5 \mu \mathrm{M}$, which was calculated based on the Max-Chelator programme (Stanford University, Stanford, $\mathrm{CA}$ ), in which the calcium dissociation constant of EGTA is $0.15 \mu \mathrm{M}^{58,59}$.

The holding potential was $-80 \mathrm{mV}$. For Train ${ }_{2 \mathrm{~Hz}}$ stimulation, a Train of $50 \mathrm{~ms}$ depolarization from -80 to $+10 \mathrm{mV}$ was given at $2 \mathrm{~Hz}$; each $50 \mathrm{~ms}$ depolarization was preceded by a $50 \mathrm{~ms}$ pre-pulse from -80 to $+120 \mathrm{mV}$, to facilitate $\mathrm{Ca}^{2+}$ channel current ${ }^{60-62}$. Cell membrane capacitance was simultaneously recorded $15 \mathrm{~s}$ before, during and $30 \mathrm{~s}$ after depolarization. The sample interval for current recordings was $50 \mu \mathrm{s}$. The frequency of the sinusoidal stimulus was $1,000-1,500 \mathrm{~Hz}$ with a peak-to-peak voltage $\leq 50 \mathrm{mV}$.

Confocal imaging. Alexa 647 (A647; 20-30 $\mu \mathrm{M}$ in bathing solution, Invitrogen) and Alexa 488 dyes (A488; $20-30 \mu \mathrm{M}$ in bathing solution, Invitrogen) were excited by a solid-state $638 \mathrm{~nm}$ ( $30 \mathrm{~mW}$ output) and $488 \mathrm{~nm}$ lasers ( $20 \mathrm{~mW}$ output) with an inverted confocal microscope (TCS SP8, Leica, Germany; original magnification, $\times 63 / 1.40$ oil objective). Unless mentioned otherwise, the $638 \mathrm{~nm}$ laser was set at $20-25 \%$ of the maximum power and the $488 \mathrm{~nm}$ laser was set at $0.5-2 \%$ of the maximum power. A647 fluorescence was collected with a photomultiplier at 639-767 nm, whereas A488 was collected with a hybrid GaAsP spectral detector at 489-596 nm. For time-lapse A647/A488 imaging, images were collected with $40 \mathrm{~ms}$ inter-frame interval at $45-70 \mathrm{~nm}$ per pixel in an imaging area of $\sim 160-320 \mu \mathrm{m}^{2}$. In some experiments, cells were pre-treated with $3 \mu \mathrm{M}$ latrunculin A (Enzo Life Sciences), $4 \mu \mathrm{M}$ Cyto D (Enzo Life Sciences), wiskostatin $(10 \mu \mathrm{M}$, Tocris Bioscience) and SMIFH2 $(25 \mu \mathrm{M}$, Tocris Bioscience) for $20 \mathrm{~min}$ in the bath solution, or whole-cell dialysis of phalloidin-FITC (1.3 $\mu \mathrm{M}$, Invitrogen) for 2-3 min before imaging.

STED imaging. The inverted STED microscopes used in this study (TCS SP5 STED, TCS SP8 STED 3X, Leica) have a resolution of $\sim 60-90 \mathrm{~nm}$. A488 $(60 \mu \mathrm{M})$ was excited with an Argon laser at $488 \mathrm{~nm}$ at $20 \%$ of the maximum power (maximum power: $25 \mathrm{~mW}$ ) and depleted with a continuous wave fibre laser at $592 \mathrm{~nm}$ using the maximum power $(1.5 \mathrm{~W})$. The fluorescence was acquired by $\mathrm{GaAsP}$ hybrid detection system at $498-580 \mathrm{~nm}$. At $20 \%$ of the maximum power, $488 \mathrm{~nm}$ laser caused A488 bleaching after pore closure with a time course similar to that of A647 under the confocal setting ${ }^{11}$. Lifeact (TagGFP2) was excited by a tunable white light laser at $470 \mathrm{~nm}$ ( $7 \%$ of maximum power) with the STED depletion laser at $592 \mathrm{~nm}$ (25\% of the maximum power) and its fluorescence at 480-560 nm was collected using time-gated detection (1.5-6.5 ns). mCherry was excited by the tunable white light laser at $570 \mathrm{~nm}$ ( $6 \%$ of maximum power) with the STED depletion laser at $660 \mathrm{~nm}$ (60\% of the maximum power) and its fluorescence between 575 and $650 \mathrm{~nm}$ was collected using time-gated detection (0.5-6.5 ns). PH-mPapaya was excited by the tunable white light laser at $530 \mathrm{~nm}(14 \%$ of the maximum power) with the STED depletion laser at $660 \mathrm{~nm}(10 \%$ of the maximum power) and its fluorescence between 541 and $620 \mathrm{~nm}$ was collected using time-gated detection (1.5-6.5 ns). When NPY-mCherry or PH-mPapaya and Lifeact (TagGFP2) were imaged at the STED microscope, NPY-mCherry (or PH-mPapaya) was imaged first, to avoid bleaching of mCherry or mPapaya fluorescence by the $592 \mathrm{~nm}$ STED depletion laser. For XZ scanning, we used $70-80 \%$ of STED depletion laser power in the $Z$-direction to improve the $z$ axis resolution.

Image analysis. A647 or A488 spots ( $\Omega$-profiles filled with A647 or A488) were identified during and $<3 \mathrm{~s}$ after the end of depolarization. The spot fluorescence intensity was measured using the LAS AF Life analysis tool or Image J and plotted using Igor Pro 6.12. The baseline fluorescence value for each spot was normalized to 1 . The representative image frames shown below the fluorescence traces were either unaveraged or averaged up to 20 frames as indicated in the figure legends. $W_{\mathrm{H}}$ was measured from intensity profiles of two to four lines across the spot centre at $45^{\circ}$ or $90^{\circ}$ apart $^{11}$

Although fusion modes ( $\Omega$-shrink fusion, stay and close fusion) were identified mostly at the cell-bottom confocal A647/A488 setting (strong/weak excitation), we sometimes identified the fusion modes with a single dye excited strongly, as described in detail in ref. 11. A single dye was used for Actb $^{-1-}$ cells overexpressed with $\beta$-Actin-mEGFP (Fig. 3b), for cells dialysed with 
phalloidin-FITC (Supplementary Fig. 3) and for STED imaging above the cell bottom (Supplementary Fig. 1c; $592 \mathrm{~nm}$ laser for depletion).

Generation of chromaffin cell-specific $\boldsymbol{\beta}$-actin KO mice. Global KO of $\beta$-actin is embryonically lethal ${ }^{63,64}$. Accordingly, we used a chromaffin cell-specific Cre mouse, the PNMT ${ }^{\mathrm{Cre} /}+{ }_{\text {mouse }}{ }^{19}$, to knock out $\beta$-actin tissue specifically in chromaffin cells. We bred the 129/SvImJ PNMTCre/ + mice with C57BL/6 Actb $^{\text {LoxPLloxP }}$ mice (Actb gene encodes $\beta$-actin ${ }^{20}$ and subsequently bred $\mathrm{PNMT}^{\mathrm{Cre} /+} ; \mathrm{Actb}^{\mathrm{LoxP} /+}$ mice with PNMT $\mathrm{Pre}^{\mathrm{C}++} ; \mathrm{Actb}^{\mathrm{LoxP} /+}$ mice to generate $\mathrm{PNMT}^{\mathrm{Cre} / \mathrm{Cre}} ; \mathrm{Actb}^{\mathrm{LoxP} / \mathrm{LoxP}}$ mice (Actb ${ }^{-1-}$ mice). Mouse genotypes were determined by PCR

Mouse chromaffin cell culture. Animal care and use were carried out in accordance with NIH guidelines and approved by NINDS/NIDCD/NCCIH Animal Care and Use Committee in NIH. Five to 10 weeks old female or male mouse adrenal glands were used for experiments to obtain chromaffin cells as described previously ${ }^{65}$ with some modification. Briefly, adrenal glands were removed from mice (two per culture) and bathed in a dissociation buffer containing (in $\mathrm{mM}$ ): 80 Na-glutamate, $55 \mathrm{NaCl}, 6 \mathrm{KCl}, 1 \mathrm{MgCl}_{2}, 10 \mathrm{HEPES}$ and $10 \mathrm{D}$-glucose $\mathrm{pH} 7$ adjusted with $\mathrm{NaOH}$. Under a dissecting microscope, adrenal cortex was carefully removed to expose the medulla. The medulla was first digested in the dissociation buffering containing papain $\left(30 \mathrm{U} \mathrm{ml}^{-1}\right.$, Sigma), BSA $\left(0.5 \mathrm{mg} \mathrm{ml}^{-1}\right.$, Sigma) and dithiothreitol $\left(0.1 \mathrm{mM}\right.$, Sigma) at $37^{\circ} \mathrm{C}$ for $8 \mathrm{~min}$ and further digested in the dissociation buffer containing collagenase $\mathrm{F}\left(3 \mathrm{U} \mathrm{ml}^{-1}\right.$, Sigma) and $\mathrm{CaCl}_{2}$ $(0.1 \mathrm{mM})$ at $37^{\circ} \mathrm{C}$. The digested medulla was carefully resuspended in DMEM medium (Invitrogen) containing 10\% fetal bovine serum (Gibco) and centrifuged at 500 r.p.m. for $3 \mathrm{~min}$. For rescue experiments, $\mathrm{Actb}^{-1-}$ cell pellet was transfected with $\beta$-Actin-mEGFP $(2 \mu \mathrm{g}$, Addgene) using a basic neuron nucleofector kit (Lonza, Program O-005). For most other experiments, cell pellet was resuspended in the fresh pre-warmed DMEM and plated onto poly-D-lysine and laminin I-coated $25 \mathrm{~mm}$-diameter glass coverslips (Neuvitro Corp.). Cells were incubated at $37^{\circ} \mathrm{C}$ with $8 \% \mathrm{CO}_{2}$ for at least $48 \mathrm{~h}$ before using for imaging or immunocytochemistry. For immunocytochemistry, cultured cells were briefly washed with $1 \times$ PBS and fixed with $4 \%$ paraformaldehyde (Electron Microscopy Sciences, USA) in PBS for $20 \mathrm{~min}$. Cells were permeabilized with $0.5 \%$ Triton $\mathrm{X}-100$ for $10 \mathrm{~min}$, blocked with $5 \%$ goat serum before incubation with mouse anti- $\beta$-actin $(1 / 500, A b c a m)$ and rabbit anti-Cre-recombinase antibodies $(1 / 250$, Abcam) in PBS containing $0.1 \%$ Triton X-100 and $1 \%$ BSA at $4{ }^{\circ} \mathrm{C}$ overnight. After washing with PBS, cells were incubated with Atto-488- and Alexa-647-conjugated secondary antibodies $(1 / 400)$ in PBS containing $1 \%$ BSA, washed with PBS and mounted. Images were examined by Zeiss LSM 510 meta confocal microscope. Same image settings were applied to both control (WT or Actb ${ }^{\text {LoxP/LoxP }}$ ) and $\beta$-actin KO mice chromaffin cells.

Micropipette aspiration measurements. A home-built micropipette aspiration system with a graduated manometer was used for application of controlled suction pressure onto living cells. Glass micropipettes with an inner diameter of $2-3 \mu \mathrm{m}$ were filled with $0.2 \% \mathrm{BSA}$ in $1 \times \mathrm{PBS}$, to allow smooth movement of the cell membrane inside the pipette. Negative pressure in the micropipette tip was generated by aspirating water from the manometer reservoir and increased gradually in $200 \mathrm{~Pa}$ increments. Nikon Advanced Modulation Contrast optics mounted on a Nikon Ti-S microscope and CoolSnap MYOCCD camera (Photometrics, Tucson, $\mathrm{AZ}$ ) were used to acquire live-cell bright-field images. Image J (http://rsb.info. nih.gov/ij/) software was used to manually measure the pipette diameters and plasma membrane projection length within the pipette.

Atomic force microscopy. AFM imaging was performed on a BioScope Catalyst II AFM with a Nanoscope $\mathrm{V}$ controller (Bruker, CA). A polystyrene microsphere with known diameter of $9.6 \mu \mathrm{m}$ (Interfacial Dynamics Corp., Portland, OR, USA) was glued onto a tipless cantilever (MLCTO10-D, Bruker) whose spring constant is calibrated by the standard thermal fluctuation method. The cell volume was obtained through the force volume mode in a scan range of $40 \times 40 \mu \mathrm{m}$ with $64 \times 64$ pixels. The volume of the same cell was measured in control, in a hyper-osmotic solution and in a hypo-osmotic solution under the same probe and parameter setting for direct comparison. The resulting topographic images were exported to ImageJ (version $1.49 \mathrm{~d}$, NIH, Bethesda, MD) for three-dimensional visualization and cell-shape computation, and for final volume ratio statistics and plotting in Microsoft Excel (version 14, Richmond, WA).

Data collection and statistical analysis in chromaffin cells. All data at the whole-cell configuration were collected within $3 \mathrm{~min}$ after whole-cell break-in, which minimized whole-cell rundown of endocytosis ${ }^{11,66}$. All experiments in chromaffin cells were carried out from at least three independent cultures. Unpaired or paired Student's $t$-test (two-tailed) was used for the statistical analysis. The data were expressed as mean \pm s.e.m. or mean + s.e.m.

Lamprey synapse and EM. Animal care and use were carried out in accordance with Karolinska Institutet guidelines and approved by the Swedish Board of
Agriculture. Dissection of the trunk region of the lamprey spinal cord, microinjection procedures, stimulation and fixation were performed as described before $^{67}$. Giant axons were stimulated at $5 \mathrm{~Hz}$ for $20-30 \mathrm{~min}$ using an extracellular electrode. Action potentials in giant axons were monitored with an extracellular suction electrode placed at the caudal part of the preparation (Supplementary Fig. 12f). The microinjection pipettes contained latrunculin $A(60 \mu \mathrm{M})$ and Cyto D $(33 \mu \mathrm{M})$, phalloidin-Alexa Flour $488\left(1,200 \mathrm{U}^{-1} \mathrm{l}^{-1}\right.$; Invitrogen), PxA $\left(5 \mathrm{mg} \mathrm{ml}^{-1}\right)$ and bovine profilin I $\left(5 \mathrm{mg} \mathrm{ml}^{-1}\right)$ in a buffer solution containing $10 \mathrm{mM}$ HEPES (pH 7.4), $250 \mathrm{mM} \mathrm{K}^{+}$acetate and $0.5 \%$ dimethyl sulfoxide. Covalently cross-linked PxA was prepared as described previously ${ }^{33}$. Compounds were diluted in Texas Red (3,000 Mw, Molecular Probes) and monitored with a charge-coupled device camera (Princeton Instruments) connected to a fluorescence microscope and a Nikon D-eclipse C1 confocal microscope using a $\times 10$ air or $\times 40$ waterimmersion objective (numerical aperture 0.80 ).

Synapses were studied for EM at distances larger than $100 \mu \mathrm{m}$ from the site of microinjections where the Texas Red fluorescence was 10-20 times lower than at the site of microinjection (Supplementary Fig. 12a). We therefore estimated that the concentration of Lat A and Cyto D at the site of EM examination was $<6$ and $3.3 \mu \mathrm{M}$, respectively.

Spinal cords were fixed during stimulation with $3 \%$ glutaraldehyde, $0.5 \%$ paraformaldehyde in $0.1 \mathrm{M}$ cacodylate buffer ( $\mathrm{pH} 7.4$ ) containing $4 \%$ tannic acid for $1 \mathrm{~h}$, followed by the same fixative without tannic acid for $3 \mathrm{~h}$. After post fixation in $1 \%$ osmium tetroxide for $1 \mathrm{~h}$ and dehydration in alcohol, the specimens were embedded in Durcupan ACM (Fluka). Serial ultrathin sections were cut with a diamond knife (Diatome) and viewed at $80 \mathrm{kV}$ in a Tecnai 12 electron microscope (FEI).

Effects of microinjections were analyzed in synapses cut in serial ultrathin sections. Samples were blinded before imaging and analysis. All described effects were reproduced in at least two independent preparations in which one to six axons were microinjected. Quantifications of fusion intermediates and synaptic vesicles at the active zone were done from serial sections of five synapses. Significances and $P$-values were calculated using one-way analysis of variance followed by Holm-Šídák's multiple comparisons test (GraphPad Prism 6).

For immunogold labelling experiments, non-stimulated lamprey spinal cords were dissected, fixed in $3 \%$ paraformaldehyde and $0.5 \%$ glutaraldehyde in $0.1 \mathrm{M}$ cacodylate buffer ( $\mathrm{pH} 7.4$ ) containing $4 \%$ tannic acid for $1 \mathrm{~h}$ at $4{ }^{\circ} \mathrm{C}$, followed by the same fixative without tannic acid for $3 \mathrm{~h}$. Specimens were stained en bloc with $2 \%$ uranyl acetate, dehydrated in graded ethanol series and embedded at $-25^{\circ} \mathrm{C}$ in LR Gold resin (Fluka). Serial ultrathin sections were collected on nickel mesh grids and incubated overnight at $4{ }^{\circ} \mathrm{C}$ with primary monoclonal actin antibodies ( $\beta$-actin Antibody (C4): sc-47778; Santa Cruz Biotech) diluted 1:100 in Tris-PBS $(\mathrm{pH} 7.4)$ containing $1 \%$ human serum albumin and $5 \%$ BSA. Secondary antibodies conjugated to $5 \mathrm{~nm}$ colloidal gold (AuroProbe EM GAM G5: RPN 424; Amersham Biosciences) were used at a dilution of 1:25. Gold particles were enhanced using the IntenSE Silver enhancement kit (RPN 492; Amersham Biosciences) following manufacturer's instructions. Sections were counterstained with uranyl acetate and lead citrate, and examined in a Tecnai 12 electron microscope (FEI). Experiments where primary antibodies were omitted did not produce any specific labelling. The number of particles was quantified in selected regions (see Supplementary Fig. 13a) and the density of gold particles per $\mu \mathrm{m}^{2}$ was calculated. Synapse was defined as a region containing synaptic vesicles clustered at dense projections at the presynaptic membrane, the active zone.

Data availability. The authors declare that all data supporting the findings of this study are available within the article and its Supplementary Information files or are available from the corresponding authors and the first authors upon request.

\section{References}

1. Alabi, A. A. \& Tsien, R. W. Perspectives on kiss-and-run: role in exocytosis, endocytosis, and neurotransmission. Annu. Rev. Physiol. 75, 393-422 (2013).

2. Wu, L. G., Hamid, E., Shin, W. \& Chiang, H. C. Exocytosis and endocytosis: modes, functions, and coupling mechanisms. Annu. Rev. Physiol. 76, 301-331 (2014).

3. Saheki, Y. \& De Camilli, P. Synaptic vesicle endocytosis. Cold Spring Harb. Perspect. Biol. 4, a005645 (2012).

4. Jahn, R. \& Fasshauer, D. Molecular machines governing exocytosis of synaptic vesicles. Nature 490, 201-207 (2012).

5. Sokac, A. M., Co, C., Taunton, J. \& Bement, W. Cdc42-dependent actin polymerization during compensatory endocytosis in Xenopus eggs. Nat. Cell Biol. 5, 727-732 (2003).

6. Nightingale, T. D. et al. Actomyosin II contractility expels von Willebrand factor from Weibel-Palade bodies during exocytosis. J. Cell Biol. 194, 613-629 (2011).

7. Masedunskas, A. et al. Role for the actomyosin complex in regulated exocytosis revealed by intravital microscopy. Proc. Natl Acad. Sci. USA 108, 13552-13557 (2011).

8. Nemoto, T., Kojima, T., Oshima, A., Bito, H. \& Kasai, H. Stabilization of exocytosis by dynamic F-actin coating of zymogen granules in pancreatic acini. J. Biol. Chem. 279, 37544-37550 (2004). 
9. Miklavc, P., Wittekindt, O. H., Felder, E. \& Dietl, P. Ca2 +-dependent actin coating of lamellar bodies after exocytotic fusion: a prerequisite for content release or kiss-and-run. Ann. N. Y. Acad. Sci. 1152, 43-52 (2009).

10. Nightingale, T. D., Cutler, D. F. \& Cramer, L. P. Actin coats and rings promote regulated exocytosis. Trends Cell Biol. 22, 329-337 (2012).

11. Chiang, H. C. et al. Post-fusion structural changes and their roles in exocytosis and endocytosis of dense-core vesicles. Nat. Commun. 5, 3356 (2014).

12. Morton, W. M., Ayscough, K. R. \& McLaughlin, P. J. Latrunculin alters the actin-monomer subunit interface to prevent polymerization. Nat. Cell Biol. 2, 376-378 (2000).

13. Brown, S. S. \& Spudich, J. A. Mechanism of action of cytochalasin: evidence that it binds to actin filament ends. J. Cell Biol. 88, 487-491 (1981).

14. Casella, J. F., Flanagan, M. D. \& Lin, S. Cytochalasin D inhibits actin polymerization and induces depolymerization of actin filaments formed during platelet shape change. Nature 293, 302-305 (1981).

15. Peterson, J. R. \& Golemis, E. A. Autoinhibited proteins as promising drug targets. J. Cell Biochem. 93, 68-73 (2004).

16. Rizvi, S. A. et al. Identification and characterization of a small molecule inhibitor of formin-mediated actin assembly. Chem. Biol. 16, 1158-1168 (2009).

17. Korn, E. D., Carlier, M. F. \& Pantaloni, D. Actin polymerization and ATP hydrolysis. Science 238, 638-644 (1987).

18. Cheever, T. R. \& Ervasti, J. M. Actin isoforms in neuronal development and function. Int. Rev. Cell Mol. Biol. 301, 157-213 (2013).

19. Ebert, S. N. et al. Targeted insertion of the Cre-recombinase gene at the phenylethanolamine n-methyltransferase locus: a new model for studying the developmental distribution of adrenergic cells. Dev. Dyn. 231, 849-858 (2004).

20. Perrin, B. J., Sonnemann, K. J. \& Ervasti, J. M. beta-actin and gamma-actin are each dispensable for auditory hair cell development but required for Stereocilia maintenance. PLoS Genet. 6, e1001158 (2010).

21. Lomasney, J. W. et al. Phosphatidylinositol 4,5-bisphosphate binding to the pleckstrin homology domain of phospholipase C-deltal enhances enzyme activity. J Biol. Chem. 271, 25316-25326 (1996).

22. Gasman, S. et al. Regulated exocytosis in neuroendocrine cells: a role for subplasmalemmal Cdc42/N-WASP-induced actin filaments. Mol. Biol. Cell 15, 520-531 (2004).

23. Wen, P. J. et al. Phosphatidylinositol $(4,5)$ bisphosphate coordinates actin-mediated mobilization and translocation of secretory vesicles to the plasma membrane of chromaffin cells. Nat. Commun. 2, 491 (2011).

24. Gabel, M. et al. Annexin A2-dependent actin bundling promotes secretory granule docking to the plasma membrane and exocytosis. J. Cell Biol. 210, 785-800 (2015)

25. Boulant, S., Kural, C., Zeeh, J. C., Ubelmann, F. \& Kirchhausen, T. Actin dynamics counteract membrane tension during clathrin-mediated endocytosis. Nat. Cell Biol. 13, 1124-1131 (2011).

26. Diz-Munoz, A. et al. Control of directed cell migration in vivo by membraneto-cortex attachment. PLoS Biol. 8, e1000544 (2010).

27. Lee, L. M. \& Liu, A. P. A microfluidic pipette array for mechanophenotyping of cancer cells and mechanical gating of mechanosensitive channels. Lab Chip 15, 264-273 (2015)

28. Tsujita, K., Takenawa, T. \& Itoh, T. Feedback regulation between plasma membrane tension and membrane-bending proteins organizes cell polarity during leading edge formation. Nat. Cell Biol. (2015).

29. Tan, X., Heureaux, J. \& Liu, A. P. Cell spreading area regulates clathrin-coated pit dynamics on micropatterned substrate. Integr. Biol. 7, 1033-1043 (2015).

30. Shupliakov, O. et al. Impaired recycling of synaptic vesicles after acute perturbation of the presynaptic actin cytoskeleton. Proc. Natl Acad. Sci. USA 99, 14476-14481 (2002).

31. Bourne, J., Morgan, J. R. \& Pieribone, V. A. Actin polymerization regulates clathrin coat maturation during early stages of synaptic vesicle recycling at lamprey synapses. J. Comp. Neurol. 497, 600-609 (2006).

32. Hajkova, L., Nyman, T., Lindberg, U. \& Karlsson, R. Effects of cross-linked profilin:beta/gamma-actin on the dynamics of the microfilament system in cultured cells. Exp. Cell Res. 256, 112-121 (2000).

33. Nyman, T., Page, R., Schutt, C. E., Karlsson, R. \& Lindberg, U. A cross-linked profilin-actin heterodimer interferes with elongation at the fast-growing end of F-actin. J. Biol. Chem. 277, 15828-15833 (2002).

34. Grenklo, S. et al. A crucial role for profilin-actin in the intracellular motility of Listeria monocytogenes. EMBO Rep. 4, 523-529 (2003).

35. Bloom, O. et al. Colocalization of synapsin and actin during synaptic vesicle recycling. J. Cell Biol. 161, 737-747 (2003).

36. Evergren, E. et al. Intersectin is a negative regulator of dynamin recruitment to the synaptic endocytic zone in the central synapse. J. Neurosci. 27, 379-390 (2007).

37. Kasai, H., Takahashi, N. \& Tokumaru, H. Distinct initial SNARE configurations underlying the diversity of exocytosis. Physiol. Rev. 92, 1915-1964 (2012).

38. Lindau, M. \& Alvarez de Toledo, G. The fusion pore. Biochim. Biophys. Acta 164, 167-173 (2003).
39. Sudhof, T. C. The synaptic vesicle cycle. Annu. Rev. Neurosci. 27, 509-547 (2004).

40. Jackson, M. B. \& Chapman, E. R. The fusion pores of $\mathrm{Ca}(2+)$-triggered exocytosis. Nat. Struct. Mol. Biol. 15, 684-689 (2008).

41. Heuser, J. E. Review of electron microscopic evidence favouring vesicle exocytosis as the structural basis for quantal release during synaptic transmission. Quart. J. Exp. Physiol. 74, 1051-1069 (1989).

42. Heuser, J. E. \& Reese, T. S. Structural changes after transmitter release at the frog neuromuscular junction. J. Cell Biol. 88, 564-580 (1981).

43. Watanabe, S. et al. Ultrafast endocytosis at Caenorhabditis elegans neuromuscular junctions. Elife 2, e00723 (2013).

44. Malacombe, M., Bader, M. F. \& Gasman, S. Exocytosis in neuroendocrine cells: new tasks for actin. Biochim. Biophys. Acta 1763, 1175-1183 2006.

45. Porat-Shliom, N., Milberg, O., Masedunskas, A. \& Weigert, R. Multiple roles for the actin cytoskeleton during regulated exocytosis. Cell Mol. Life Sci. 70, 2099-2121 (2013).

46. Berberian, K., Torres, A. J., Fang, Q., Kisler, K. \& Lindau, M. F-actin and myosin II accelerate catecholamine release from chromaffin granules. $J$. Neurosci. 29, 863-870 (2009).

47. Neco, P. et al. Myosin II contributes to fusion pore expansion during exocytosis. J. Biol. Chem. 283, 10949-10957 (2008).

48. Olivares, M. J. et al. Src kinases regulate de novo actin polymerization during exocytosis in neuroendocrine chromaffin cells. PLoS ONE 9, e99001 (2014).

49. Fang, Q. et al. The role of the C terminus of the SNARE protein SNAP-25 in fusion pore opening and a model for fusion pore mechanics. Proc. Natl Acad. Sci. USA 105, 15388-15392 (2008).

50. Doreian, B. W., Fulop, T. G. \& Smith, C. B. Myosin II activation and actin reorganization regulate the mode of quantal exocytosis in mouse adrenal chromaffin cells. J. Neurosci. 28, 4470-4478 (2008).

51. Klyachko, V. A. \& Jackson, M. B. Capacitance steps and fusion pores of small and large-dense-core vesicles in nerve terminals. Nature 418, 89-92 (2002).

52. He, L., Wu, X. S., Mohan, R. \& Wu, L. G. Two modes of fusion pore opening revealed by cell-attached recordings at a synapse. Nature 444, 102-105 (2006).

53. Rangaraju, V., Calloway, N. \& Ryan, T. A. Activity-driven local ATP synthesis is required for synaptic function. Cell 156, 825-835 (2014).

54. Hroudova, J., Singh, N. \& Fisar, Z. Mitochondrial dysfunctions in neurodegenerative diseases: relevance to Alzheimer's disease. Biomed. Res. Int. 2014, 175062 (2014).

55. Lian, G. \& Sheen, V. L. Cytoskeletal proteins in cortical development and disease: actin associated proteins in periventricular heterotopia. Front. Cell Neurosci. 9, 99 (2015).

56. Lindau, M. \& Neher, E. Patch-clamp techniques for time-resolved capacitance measurements in single cells. Pflugers Arch. 411, 137-146 (1988).

57. Heidelberger, R. ATP is required at an early step in compensatory endocytosis in synaptic terminals. J. Neurosci. 21, 6467-6474 (2001).

58. Augustine, G. J. \& Neher, E. Calcium requirements for secretion in bovine chromaffin cells. J. Physiol. 450, 247-271 (1992).

59. Elhamdani, A., Azizi, F. \& Artalejo, C. R. Double patch clamp reveals that transient fusion (kiss-and-run) is a major mechanism of secretion in calf adrenal chromaffin cells: high calcium shifts the mechanism from kiss-and-run to complete fusion. J. Neurosci. 26, 3030-3036 (2006).

60. Artalejo, C. R., Adams, M. E. \& Fox, A. P. Three types of $\mathrm{Ca}^{2+}$ channel trigger secretion with different efficacies in chromaffin cells. Nature 367, 72-76 (1994).

61. Artalejo, C. R., Henley, J. R., McNiven, M. A. \& Palfrey, H. C. Rapic endocytosis coupled to exocytosis in adrenal chromaffin cells involves $\mathrm{Ca}^{2+}, \mathrm{GTP}$, and dynamin but not clathrin. Proc. Natl Acad. Sci. USA 92, 8328-8332 (1995).

62. Artalejo, C. R., Elhamdani, A. \& Palfrey, H. C. Calmodulin is the divalent cation receptor for rapid endocytosis, but not exocytosis, in adrenal chromaffin cells. Neuron 16, 195-205 (1996).

63. Shawlot, W., Deng, J. M., Fohn, L. E. \& Behringer, R. R. Restricted beta-galactosidase expression of a hygromycin-lacZ gene targeted to the beta-actin locus and embryonic lethality of beta-actin mutant mice. Transgenic Res. 7, 95-103 (1998).

64. Shmerling, D. et al. Strong and ubiquitous expression of transgenes targeted into the beta-actin locus by Cre/lox cassette replacement. Genesis 42, 229-235 (2005).

65. Wu, X. S. et al. Calcineurin is universally involved in vesicle endocytosis at neuronal and nonneuronal secretory cells. Cell Rep. 7, 982-988 (2014).

66. Smith, C. \& Neher, E. Multiple forms of endocytosis in bovine adrenal chromaffin cells. J. Cell Biol. 139, 885-894 (1997).

67. Gad, H. et al. Fission and uncoating of synaptic clathrin-coated vesicles are perturbed by disruption of interactions with the $\mathrm{SH} 3$ domain of endophilin. Neuron 27, 301-312 (2000).

\section{Acknowledgements}

We thank Dr James Ervasti for providing Actbloxp mice. This work was supported by the National Institute of Neurological Disorders and Stroke Intramural Research Program (ZIA NS003009-13 and ZIA NS003105-08) to L.G.W. and by grants from the Swedish 
Research Council (project 13473), Parkinsonfonden, Hjärnfonden and the Russian Science Foundation (project number 16-15-10273) to O.S.

\section{Author contributions}

P.J.W. and S.G. did most experiments in chromaffin cells and lamprey synapses, respectively. G.A., S.Y.P., H.C.C., E.H., W.D.Z., W.S., T.N., E.E., Y.J., R.K. and S.N.E. participated in experiments. R.K. provided PxA. H.S.L. and A.J. performed atomic force microscopy. X.T., J.H. and A.P.L. performed micropipette aspiration to measure membrane elasticity. L.G.W. and O.S. conceived and designed experiments for chromaffin cells and lamprey synapses, and wrote the manuscript with help from P.J.W.

\section{Additional information}

Supplementary Information accompanies this paper at http://www.nature.com/ naturecommunications

Competing financial interests: The authors declare no competing financial interests.
Reprints and permission information is available online at http://npg.nature.com/ reprintsandpermissions/

How to cite this article: Wen, P. J. et al. Actin dynamics provides membrane tension to merge fusing vesicles into the plasma membrane. Nat. Commun. 7:12604 doi: $10.1038 /$ ncomms12604 (2016).

\section{(c) ()}

This work is licensed under a Creative Commons Attribution 4.0 International License. The images or other third party material in this article are included in the article's Creative Commons license, unless indicated otherwise in the credit line; if the material is not included under the Creative Commons license, users will need to obtain permission from the license holder to reproduce the material To view a copy of this license, visit http://creativecommons.org/licenses/by/4.0/

(C) The Author(s) 2016 